\title{
STABILITY OF MULTI-LAYER CELLULAR NEURAL/NONLINEAR NETWORKS
}

\author{
LEVENTE TÖRÖK \\ Analogic and Neural Computing Laboratory, Computer and Automation Research Institute, \\ Hungarian Academy of Sciences, H-1111, Budapest, Kende u. 13-17, Hungary \\ torok@sztaki.hu \\ TAMÁS ROSKA \\ Faculty of Information Technology, Pázmány Péter Catholic University, \\ H-1052, Budapest, Piarista köz 1., Hungary \\ roska@sztaki.hu
}

Received August 19, 2003; Revised October 30, 2003

\begin{abstract}
We have found a formalism that lets us present generalizations of several stability theorems (see Chua \& Roska, 1990; Chua \& Wu, 1992; Gilli, 1993; Forti, 2002] on Multi-Layer Cellular Neural/Nonlinear Networks (MLCNN) formerly claimed for Single-Layer Cellular Neural/Nonlinear Networks (CNN). The theorems were selected with special regard to usefulness in engineering applications. Hence, in contrast to many works considering stability on recurrent neural networks, the criteria of the new theorems have clear indications that are easy to verify directly on the template values. Proofs of six new theorems on 2-Layer CNNs (2LCNN) related to symmetric, $\tau$-symmetric, nonsymmetric, $\tau$-nonsymmetric, and sign-symmetric cases are given. Furthermore, a theorem with a proof on a MLCNN with arbitrary template size and arbitrary layer number in relation to the sign-symmetric theorem is given, along with a conjecture for the one-dimensional, two-layer, nonreciprocal case.
\end{abstract}

Keywords: Multi-layer CNN; recurrent network stability; CACE.

\section{Introduction}

The analysis of the stability of Multi-Layer Cellular Neural/Nonlinear Networks (MLCNN) [Chua \& Yang, 1988b; Chua \& Roska, 1990] is motivated by the appearance of a new 2-Layer CNN Universal Machine (CNN-UM) architecture called CACE (Complex cell Analog-and-logic non-linear Computing Engine) [Carmona et al., 2002], that has already been manufactured. Interestingly, the region of stable operation (i.e. template values) of Multi-Layer architetures' has not been analyzed as intimately as its Single-Layer counterpart which is still far from being entirely clarified yet.

However they are not completely discovered yet, several important spatio-temporal phenomena have been experimentally tested and its relevance in modeling has been demonstrated [Rekeczky et al., 2000].

The main virtue of our paper is a formalism that enables us to treat Multi-Layer architectures as simple as a Single-Layer CNN.

The main results refer mostly to a 2-Layer CNN. Namely, we show that a $2 \mathrm{LCNN}$ is stable

- if the inter-layer feedback templates are symmetric, and the intra-layer feedback template is crosssymmetric or $\tau$-cross symmetric (i.e. weighted with the corresponding layer time constant),

- if in the class of nonsymmetric templates, the so-called constant-ratio rule holds among the template entries, 
- if all the templates match to a single sign pattern in which sign alteration frequency is a fixed one or zero in both horizontal and vertical directions and the phases of the two inter-layer feedbacks are equivalent while the inter-layer feedbacks may vary upto a constant phase compared to them.

Similarly MLCNN with arbitrary layer number is stable with arbitrary template size if a similar hypothesis holds with the extension that the matrix of the phase changes is in the class of chess board type matrices.

In the following, the CACE architecture is described step-by-step (Sec. 2) starting from the Chua Yang model of CNN, then its compact vector form is reviewed with the extension to multi-layer equivalent equations that made the generalization of the formerly known theorems possible (Sec. 3), and new theorems are presented in Sec. 4. The appendix recalls the known and the new proofs with our unified formalism to help the reader, however our contribution can be found in the main part.

\section{Multi-Layer and CACE Architectures}

\subsection{Chua-Yang model}

A CNN [Chua \& Yang, 1988b] operates on a lattice-structured set of state variables $(\hat{x})_{i j}, i \in$ $\{1, \ldots, N\} ; j \in\{1, \ldots, M\}^{1}$ input $\left(\hat{u}_{i j}\right)$ and bias map $\left(\hat{z}_{i j}\right)$ influenced by each other via a small set of local connection variables, called template ( $\hat{A}$ and $\hat{B})$. Both the variables and the templates are arranged in matrix form. In hardware implementations the size of the templates usually does not extend beyond the neighborhood of radius $r=1$, which mean a $3 \times 3$ template size (i.e. $r=1 \Leftrightarrow$ $\left.\exists(\hat{A})_{k l} \neq 0: k, l \in\{-1,0,1\}\right)$.

The system is governed by the following differential equation,

$$
\begin{aligned}
\tau(\dot{\hat{x}}(t))_{i j}= & -(\hat{x}(t))_{i j}+\sum_{k l \in S_{r}(i j)}(\hat{A})_{k l} \cdot(\hat{y}(t))_{i+k, j+l} \\
& +\sum_{k l \in S_{r}(i j)}(\hat{B})_{k l} \cdot(\hat{u}(t))_{i+k, j+l}+(\hat{z}(t))_{i j}
\end{aligned}
$$

or in short

$$
\tau \dot{\hat{x}}=-\hat{x}+\hat{A} * \hat{y}+\hat{B} * \hat{u}+\hat{z}
$$

where $*$ symbolizes the convolution operator. Note that capitals are constants.

$$
\begin{aligned}
(\hat{y})_{i j} & =f\left((\hat{x})_{i j}\right) \\
& =\frac{\left(\left|(\hat{x})_{i j}+c\right|-\left|(\hat{x})_{i j}-c\right|\right)}{2 c}
\end{aligned}
$$

where $c$ is usually chosen to be 1 .

This model was introduced by Chua and Yang [1988b] therefore it is called Chua-Yang model (CYCNN).

\subsection{Full-range model}

For practical reasons, different types of nonlinearities were introduced. One of them is the so-called Full-range model [Espejo et al., 1994], which limits the operational range of state variables $(\hat{x})_{i j}$, rather than limiting the output as in Eq. (1),

$$
\tau \dot{\hat{x}}=-g(\hat{x})+\hat{A} * \hat{x}+\hat{B} * \hat{u}+\hat{z}
$$

where

$$
g\left((\hat{x})_{i j}\right)= \begin{cases}(\hat{x})_{i j}, & \text { if }\left|(\hat{x})_{i j}\right|<1 \\ \lim _{m \rightarrow \infty}\left(\left|(\hat{x})_{i j}\right|-1\right) \cdot m \cdot \mathbf{s g n}\left((\hat{x})_{i j}\right), & \text { else. }\end{cases}
$$

The function $g()$ is a limit function because of its asymptotic definition. A definition like this, indeed, imposes difficulties in numerical tractability and in functional analysis but it is easier to implement and it speeds up the operation (either convergence or instability) compared to the CY model. Furthermore, it reduces noise sensitivity in the analog implementations.
Since the majority of the stability works was made on CYCNN model and almost exclusively FRCNN model was implemented, one might ask the question whether the stability theorems claimed for CYCNN holds for FRCNN too? Some important aspects of the differences between the two models (i.e. CYCNN and FRCNN) were examined in

\footnotetext{
${ }^{1}$ We index and use brackets of matrices as $\left(m_{i j}(t)\right)_{k l}$, where $(i j)$ refers to the submatrix in the $i$ th row, $j$ th column of the hypermatrix $(m)$ at time $(t)$ and $(k l)$ refers to the matrix element in $k$ th row, $l$ th column.
} 


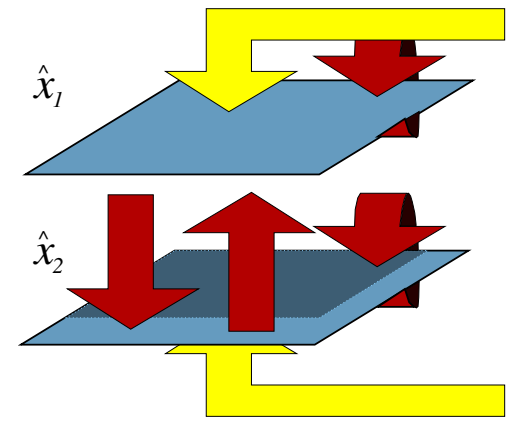

(a) General 2-layer CNN (2LCNN) architecture

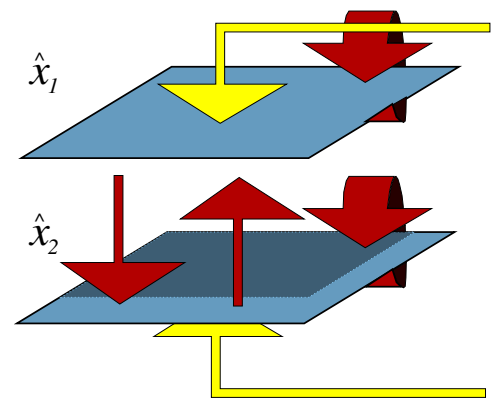

(b) The CACE architecture

Fig. 1. The fully interconnected MLCNN and the sparsely interconnected CACE architectures are depicted on the left and right, respectively. Intra-layer feedback templates $\left(\hat{A}_{11}\right.$ and $\left.\hat{A}_{22}\right)$ are symbolized by self-closing arrows on the layers $\left(\hat{x}_{1}\right.$ and $\hat{x}_{2}$ ), intra-layer feedback templates are symbolized by arrows between the layers. The differences between CACE and 2-layer architectures are emphasized by the width of the arrows referring to the size of convolution kernel.

[Cortino \& Gilli, 2003], where it was observed that even the trajectories of the two models might depart qualitatively, the convergence criteria of all the stability theorems that have been known so far are still intact.

\subsection{Multi-layer architectures}

Even though Multi-Layer CNN (MLCNN) architectures have been known since 1991 [Chua \& Shi, 1991], it did not generate as many publications as the Single-Layer architectures did, which is probably due to its increased complexity in the analysis.

Multi-layer architectures consist of two or more sets of indexed state variables influenced via $(\hat{A})_{o p}$ kernels where $o$ and $p$ refer to the so-called from and to layers, respectively. Disregarding the technical difficulties in VLSI implementations, MLCNNs could be defined as,

$$
\tau_{p} \dot{\hat{x}}_{p}=-g\left(\hat{x}_{p}\right)+\sum_{o=1}^{L} \hat{A}_{o p} * \hat{x}_{o}+\hat{B}_{p} * \hat{u}_{p}+\hat{z}_{p}
$$

where $L$ defines the number of layers $(p \in$ $\{1, \ldots, L\})$.

One should note that the entries in vector $\tau$ might vary from layer to layer. For a simple case like $L=2$ (i.e. $2 \mathrm{LCNN}$ ), one can get,

$$
\begin{aligned}
\tau_{1} \dot{\hat{x}}_{1}= & -g\left(\hat{x}_{1}\right)+\hat{A}_{11} * \hat{x}_{1}+\hat{A}_{21} * \hat{x}_{2} \\
& +\hat{B}_{1} * \hat{u}_{1}+\hat{z}_{1} \\
\tau_{2} \dot{\hat{x}}_{2}= & -g\left(\hat{x}_{2}\right)+\hat{A}_{12} * \hat{x}_{1}+\hat{A}_{22} * \hat{x}_{2} \\
& +\hat{B}_{2} * \hat{u}_{2}+\hat{z}_{2} .
\end{aligned}
$$

Defining the same architecture with respect to Chua-Yang model,

$$
\begin{aligned}
& \tau_{1} \dot{\hat{x}}_{1}=-\hat{x}_{1}+\hat{A}_{11} * \hat{y}_{1}+\hat{A}_{21} * \hat{y}_{2}+\hat{B}_{1} * \hat{u}_{1}+\hat{z}_{1} \\
& \tau_{2} \dot{\hat{x}}_{2}=-\hat{x}_{2}+\hat{A}_{12} * \hat{y}_{1}+\hat{A}_{22} * \hat{y}_{2}+\hat{B}_{2} * \hat{u}_{2}+\hat{z}_{2} .
\end{aligned}
$$

\subsection{CACE architecture}

The CACE architecture [Carmona, 2002] with a complete $\mathrm{CNN}-\mathrm{UM}$ core is defined as

$$
\begin{aligned}
\tau_{1} \dot{\hat{x}}_{1}= & -g\left(\hat{x}_{1}\right)+\hat{A}_{11} * \hat{x}_{1}+\hat{a}_{21} \cdot \hat{x}_{2} \\
& +\hat{b}_{1} \cdot \hat{u}_{1}+\hat{z}_{1} \\
\tau_{2} \dot{\hat{x}}_{2}= & -g\left(\hat{x}_{2}\right)+\hat{a}_{12} \cdot \hat{x}_{1}+\hat{A}_{22} * \hat{x}_{2} \\
& +\hat{b}_{2} \cdot \hat{u}_{2}+\hat{z}_{2} .
\end{aligned}
$$

One can define CACE architecture with respect to Chua-Yang model as

$$
\begin{aligned}
& \tau_{1} \dot{\hat{x}}_{1}=-\hat{x}_{1}+\hat{A}_{11} * \hat{y}_{1}+\hat{a}_{21} \cdot \hat{y}_{2}+\hat{b}_{1} \cdot \hat{u}_{1}+\hat{z}_{1} \\
& \tau_{2} \dot{\hat{x}}_{2}=-\hat{x}_{2}+\hat{a}_{12} \cdot \hat{y}_{1}+\hat{A}_{22} * \hat{y}_{2}+\hat{b}_{2} \cdot \hat{u}_{2}+\hat{z}_{2} .
\end{aligned}
$$

In Eq. (6) lower case $\hat{a}_{21}, \hat{a}_{12}, \hat{b}_{1}, \hat{b}_{2}$ refer to scalar values which are multiplied by the state and the input vectors, rather than convolved with them. This simplification is due to the costs of implementation belonging to the fully connected inter-layer feedback templates. Nevertheless, interesting spatio-temporal dynamics can evolve in this structure.

In Fig. 1 one can compare differences between a 2-layer and a CACE architecture.

Note that in contrast to former CNN implementations the ratio of $\tau_{1}$ and $\tau_{2}$ time constants is programmable. 


$$
\hat{A}=\begin{array}{|l|l|l|}
\hline a_{n w} & a_{n} & a_{n e} \\
\hline a_{w} & a_{c} & a_{e} \\
\hline a_{s w} & a_{s} & a_{s e} \\
\hline
\end{array}
$$

(a) Entries in the kernels are denoted by the capitals of compass points (i.e. $n$ for north, $e$ for east, etc.) plus $c$ for central.

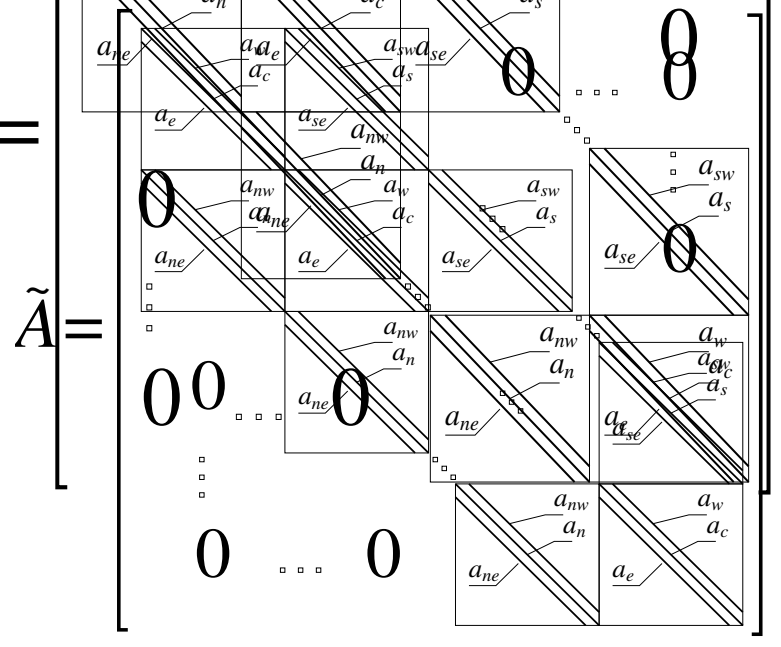

(b) The same kernel in compact matrix representation form.

Fig. 2. To transform the convolution in Eq. (1) to vector-matrix multiplication, a matrix-to-vector repacking described by Eq. (7) was used. Therefore, the matrix of kernels needed to be converted to the so-called compact matrix form having Block Toeplitz structure as shown on the right-hand side.

\section{Compact Vector Form}

At first sight, the parts most difficult to handle in the system of equations are the convolutions. To get rid of them, a standard rewriting method is used.

Consider a state equation of an $M \times N \mathrm{CNN}$. In the compact form, new vectors $(\tilde{x}, \tilde{y}, \tilde{u})$ are introduced by repacking variables by an invertible rearrangement

$$
(\tilde{x})_{i+M \cdot j}=(\hat{x})_{i j} .
$$

In order to be able to write the equivalent system of equations, the form of the kernels needs to be reformulated as well, resulting in a Block Toeplitz matrix shown in Fig. 2.

So the equivalent system of equations may be written as

$$
\tau \dot{\tilde{x}}=-g(\tilde{x})+\tilde{A} \tilde{x}+\tilde{B} \tilde{u}+\tilde{z} .
$$

Applying this to a $2 \mathrm{LCNN}$, one can write Eq. (5) as

$$
\begin{aligned}
& \tau_{1} \dot{\tilde{x}}_{1}=-g\left(\tilde{x}_{1}\right)+\tilde{A}_{11} \tilde{x}_{1}+\tilde{A}_{21} \tilde{x}_{2}+\tilde{B}_{1} \tilde{u}_{1}+\tilde{z}_{1} \\
& \tau_{2} \dot{\tilde{x}}_{2}=-g\left(\tilde{x}_{2}\right)+\tilde{A}_{12} \tilde{x}_{1}+\tilde{A}_{22} \tilde{x}_{2}+\tilde{B}_{2} \tilde{u}_{2}+\tilde{z}_{2} .
\end{aligned}
$$

By packing the state, the output, the input and the bias map vectors of the two layers in single vectors, as $x^{T}=\left(\tilde{x}_{1}^{T}, \tilde{x}_{2}^{T}\right), y^{T}=\left(\tilde{y}_{1}^{T}, \tilde{y}_{2}^{T}\right)$, etc., one can write

$$
\tau \dot{x}=-g(x)+A x+B u+z
$$

where hypermatrices $\tau, A, B$ were created by similar concatenation as

$$
\begin{gathered}
\tau=\left(\begin{array}{cc}
\tau_{1} I & 0 \\
0 & \tau_{2} I
\end{array}\right), \quad A=\left(\begin{array}{cc}
\tilde{A}_{11} & \tilde{A}_{21} \\
\tilde{A}_{12} & \tilde{A}_{22}
\end{array}\right), \\
B=\left(\begin{array}{cc}
\tilde{B}_{1} & 0 \\
0 & \tilde{B}_{2}
\end{array}\right)
\end{gathered}
$$

where $I$ denotes the identity matrix. Each hypermatix is of size $2 M N \times 2 M N$. Note that, the compact vector form of CNN is exactly the same as Eq. (10). It can easily be extended for arbitrary number of layers with arbitrary size of templates. The form still holds.

However it needs to be remarked that there is one order of magnitude difference in the degree of freedoms of the convolution kernels $\left(d f\left(\hat{A}_{i j}\right) \rightarrow\right.$ $4 \times 9)$ and their counterpart matrices $\left(d f\left(A_{i j}\right) \rightarrow\right.$ $2 M N \times 2 M N)$. Naturally, not all stable solutions for matrix $A$ can be transformed to a kernel. The main constraint on the matrix structure is the principle of spatial invariance.

The obtained form provides a powerful tool, by which many of the known theorems claimed for Single-Layer CNNs can be generalized to MLCNN architectures. All we need to do is to carefully revise all steps that might touch the degree of freedom of matrix $A$ and explore all the new possibilities inherent to its greater being. 
In the next sections we give an incomplete list of theorems that can be extended in this manner.

Definition 1. If in network none of the cell's state depends explicitly on the cell's position (or site index) then the network is called spatially invariant.

In the definition above, implicit dependence might refer to dependence on index differences.

Disregarding boundary conditions, the definition can be formulated for a Single-Layer CNNs as,

$$
(A)_{i j}=\text { function }(i-j)
$$

(read as: the entries of matrix $A$ depend exclusively on a function of the difference of the entries' horizontal and vertical indexes), which is equivalent to the definition of Toeplitz matrices. Similar constraints apply to MLCNNs,

$$
\left(A_{k l}\right)_{i j}=\operatorname{function}(k l, i-j) \text {. }
$$

Definition 2. The definition of the locality can be formulated as

$$
(\hat{A})_{i j}=0:\|i, j\|>r \Rightarrow A_{\sigma(k, l ; i, j)}=0:\|i, j\|>r
$$

where $\sigma$ is an invertible mapping of state variables from $\hat{A}$ to $A$.

Remark 1. The Bidirectional Associative Memory (BAM) neural network resembles a $2 \mathrm{LCNN}$ to a certain extent. This network was introduced by Kosko [1988] as,

$$
\begin{aligned}
& \left(\dot{\hat{x}}_{1}\right)_{i}=-\left(\kappa_{1}\right)_{i}\left(\hat{x}_{1}\right)_{i}+\sum_{j=1}^{p}\left(\hat{A}_{1}\right)_{j i} \cdot\left(\hat{y}_{2}\right)_{j}+\left(\hat{\phi}_{1}\right)_{i} \\
& \left(\dot{\hat{x}}_{2}\right)_{i}=-\left(\kappa_{2}\right)_{i}\left(\hat{x}_{2}\right)_{i}+\sum_{j=1}^{p}\left(\hat{A}_{2}\right)_{j i} \cdot\left(\hat{y}_{1}\right)_{j}+\left(\hat{\phi}_{2}\right)_{i} .
\end{aligned}
$$

which is equivalent to,

$$
\begin{aligned}
\frac{1}{\left(\kappa_{1}\right)_{i}}\left(\dot{\hat{x}}_{1}\right)_{i}= & -\left(\hat{x}_{1}\right)_{i}+\frac{1}{\left(\kappa_{1}\right)_{i}} \\
& \times\left(\sum_{j=1}^{p}\left(\hat{A}_{1}\right)_{j i} \cdot\left(\hat{y}_{2}\right)_{j}+\left(\hat{\phi}_{1}\right)_{i}\right) \\
\frac{1}{\left(\kappa_{2}\right)_{i}}\left(\dot{\hat{x}}_{2}\right)_{i}= & -\left(\hat{x}_{2}\right)_{i}+\frac{1}{\left(\kappa_{2}\right)_{i}} \\
& \times\left(\sum_{j=1}^{p}\left(\hat{A}_{2}\right)_{j i} \cdot\left(\hat{y}_{1}\right)_{j}+\left(\hat{\phi}_{2}\right)_{i}\right) .
\end{aligned}
$$

This network can be regarded as a one-dimensional, two-layer, fully connected, heteroassociative network. With the matrix concatenation formalism provided above Eq. (13) can be rewritten as,

$$
\tilde{\chi} \dot{\tilde{x}}=-\tilde{x}+\tilde{A} \tilde{y}+\tilde{\phi}
$$

where $\tilde{\chi}=\left(\begin{array}{cc}\chi_{1} & 0 \\ 0 & \chi_{2}\end{array}\right)$ composed of $\chi_{1}=$ $\operatorname{diag}\left\{1 /\left(\kappa_{1}\right)_{1}, 1 /\left(\kappa_{1}\right)_{2}, \ldots, 1 /\left(\kappa_{1}\right)_{P}\right\}$ and $\chi_{2}=$ $\left.\operatorname{diag}\left\{1 /\left(\kappa_{2}\right)_{1}, 1 /\left(\kappa_{2}\right)_{2}, \ldots, 1 /\left(\kappa_{2}\right)_{P}\right\}\right)$ denotes the neuron charging time constant, $\tilde{x}^{T}=\left(x_{1}^{T}, x_{2}^{T}\right)$ and $\tilde{A}=\left(\begin{array}{cc}0 & \chi_{1} \cdot A_{1} \\ \chi_{2} \cdot A_{2} & 0\end{array}\right)$ denotes the connection matrix. $\tilde{y}^{T}=\left(y_{1}^{T}, y_{2}^{T}\right)$ is given by $(\tilde{y})_{i}=s_{i}\left(x_{i}(t)\right)$ where $s_{i}$ represent the neural activation function with the well-known sigmoid characteristics and finally $\tilde{\phi}^{T}=\left(\left(\chi_{1} \tilde{\phi}_{1}\right)^{T},\left(\chi_{2} \tilde{\phi}_{2}\right)^{T}\right)$ is a constant additive term.

It looks clear that the only difference between 2LCNN and BAM is manifested in spatial invariance and locality criteria of $A$. Apart from this, the two networks' dynamical behavior is equivalent.

In Secs. 4.1 and 4.3 we start from the $\tau_{1}=$ $\tau_{2}=1$ case and develop toward the $\tau_{1} \neq \tau_{2}$ case in Secs. 4.2 and 4.4, while Theorem 5 is left unaffected by the modification of temporal parameters (layer time constants) but spectacular extension for larger template size is detailed in Sec. 4.5.2.

\section{Main Results}

Probably the most widely used approach for concluding systems' stability is done by means of Lyapunov functions. Sometimes it is particularly difficult to write an appropriate Lyapunov functional for a given system since its definition is not constructive. Fortunately, several Lyapunov functions have been introduced for CNN versions but other stability considerations coexist. In the following sections, several of them will be introduced.

\subsection{Symmetric case}

The root of the following theorem can be found in [Chua \& Roska, 2002] for Single-Layer CNNs, where perfect central symmetry is assumed for the template elements. Our new theorem is a logical extension to that, and proposes relation for interconnecting template elements.

Definition 3. Cross symmetry is defined as centrally mirrored equivalence of template elements in $\hat{A}_{12}$ and $\hat{A}_{21}$. [i.e. $\left(\hat{A}_{12}\right)_{k l}=\left(\hat{A}_{21}\right)_{-k-l}$ or see Fig. 3(b)]. 


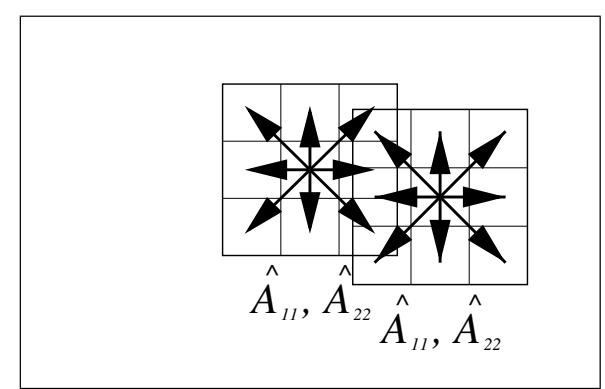

(a) First criterion: Central-symmetry must hold for intra-layer feedback template elements.

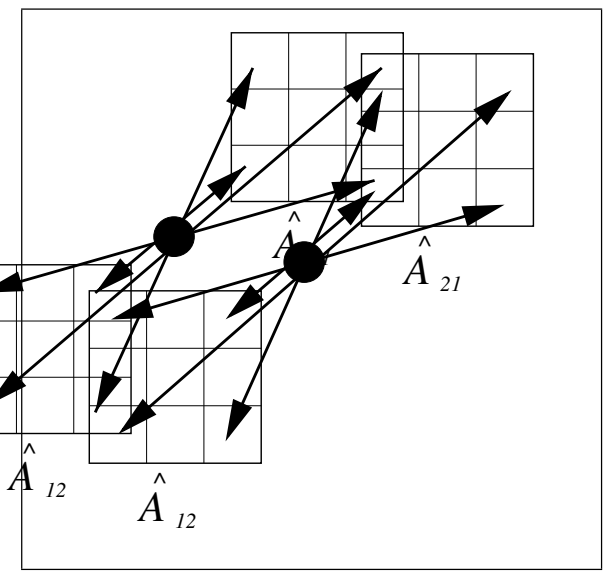

(b) Second criterion: Cross-symmetry must hold for inter-layer feedback template elements.

Fig. 3. Arrows point to kernel entries needed to be equal to satisfy the first two criteria of Theorem 1 .

Definition 4. A dynamical system $(\dot{x}=$ $\left.f(x(t)), x\left(t_{0}\right)=x_{0}, x \in \mathbb{R}^{n}, f: \mathbb{R}^{n} \rightarrow \mathbb{R}^{n}\right)$ is said to be completely stable if for $\forall x_{0} \in \mathbb{R}^{n}$ :

$$
\lim _{t \rightarrow \infty} x\left(t, x_{0}\right)=\mathrm{const}
$$

[Gilli, 1993]

Theorem 1. A 2-layer $C N N$ is completely stable $^{2}$ if

- the template values are central symmetric for intra-layer feedback sub-template elements $\left(\hat{A}_{11}, \hat{A}_{22}\right)$,

- the template values are cross symmetric for inter-layer feedback sub-template elements $\left(\hat{A}_{12}, \hat{A}_{21}\right)$,

- layer time constants are unity $\left(\tau_{1}=\tau_{2}=1\right)$,

- $f()$ is continuously differentiable, bounded and $f^{\prime}()>0$,

- all equilibrium points are isolated.

The interpretation of the criteria: The first criterion was probably the very first known by the CNN community known as central symmetry. In Fig. 3 arrows point to template elements that need to be equal to satisfy central symmetry [Fig. 3(a)] and cross symmetry [Fig. 3(b)].
The example below meets both criteria above.

$\hat{A}_{11}=$\begin{tabular}{|c|c|c|}
\hline 0.2 & 0.3 & 0.5 \\
\hline 0.4 & 2 & 0.4 \\
\hline 0.5 & 0.3 & 0.2 \\
\hline
\end{tabular}

$\hat{A}_{12}=$\begin{tabular}{|c|c|c|}
\hline 0.1 & 0.5 & 0.2 \\
\hline 0.2 & 1 & 0.2 \\
\hline 0.3 & 0.3 & 0.4 \\
\hline
\end{tabular}

$\hat{A}_{21}=$\begin{tabular}{|c|c|c|}
\hline 0.4 & 0.3 & 0.3 \\
\hline 0.2 & 1 & 0.2 \\
\hline 0.2 & 0.5 & 0.1 \\
\hline
\end{tabular}

$\hat{A}_{22}=$\begin{tabular}{|c|c|c|}
\hline 0.1 & 0.2 & 0.3 \\
\hline 0.1 & 2 & 0.1 \\
\hline 0.3 & 0.2 & 0.1 \\
\hline
\end{tabular}

Later we will recall the trajectory limit length theory of Forti et al. [2002] which enables us to drop the last two hypotheses of this theorem as well.

Proof. Assume a continuously differentiable and bounded bijective function $y(x)$, then the equilibrium points of Eq. (9) are isolated.

In the case of symmetric, spatial invariant templates, the compact matrix is symmetric $\left(A=A^{T}\right)$, which lets us write a Lyapunov function of the form,

$$
\begin{aligned}
V(x)= & -\frac{1}{2} y^{T} A y+\sum_{i=1}^{2 M N}\left[\int_{\theta}^{y_{i}} f^{-1}(v) d v\right] \\
& -y^{T} B u-y^{T} z
\end{aligned}
$$

which is the first Lyapunov function introduced for CNNs in [Chua \& Yang, 1988a].

\footnotetext{
${ }^{2}$ Here we restrict ourself to autonomous systems, hence constants $u(t)$ and $z(t)$ are assumed.
} 
In [Chua \& Yang, 1988b] it is proved that $x(t)$ is bounded $\left(\exists X_{l}:|x(t)|<X_{l}, \forall t\right)$ which means the boundedness of $V(x)$. Having a bounded $V($ ) and being its time derivative along the trajectory negative (see Appendix A), $V($ ) is an appropriate Lyapunov function of the system.

By applying LaSalle's invariant principle [LaSalle, 1967], one can conclude that all trajectories are convergent to the invariant set defined by $M=\{x: \dot{V}(x)=0\} \forall x \in \mathbb{R}^{n}$. In fact, complete stability is derived from the connectedness of the $\omega$-limit set of each trajectory.

Corollary. In the case of Single-Layer CNNs, the symmetric property of matrix $\left(A=A^{T}\right)$ implies the symmetry of the $\hat{A}$ template $\left((\hat{A})_{k l}=(\hat{A})_{-k-l}\right)$. In the case of $2 L C N N$ this property implies slightly different rules, as well.

Obviously, the symmetry is repeated for $\hat{A}_{11}$ and $\hat{A}_{22}$ intra-layer feedback templates $\left(\left(\hat{A}_{i i}\right)_{k l}=\right.$ $\left.\left(\hat{A}_{i i}\right)_{-k-l} i \in\{1,2\}\right)$, since their spatial arrangement in hypermatrix $A$ is identical to that of the original CNN from at least the symmetric point of view (i.e. diagonal matrix positions). The only difference arises in case of inter-layer feedback matrices for which the following rule can be concluded,

$$
\tilde{A}_{21}=\tilde{A}_{12}^{T} \Rightarrow\left(\hat{A}_{12}\right)_{k l}=\left(\hat{A}_{21}\right)_{-k-l} .
$$

This relation is called cross-symmetry between inter-layer feedback sub-template elements.

Remark 2. It must be noted that the case of perfectly symmetric intra-layer feedback and perfectly anti-symmetric inter-layer feedback case $\left(\left(\hat{A}_{12}\right)_{k l}=\right.$ $\left.-\left(\hat{A}_{21}\right)_{-k-l}\right)$ have already been studied in [Forti et al., 1993].

\section{2. $\tau$-Symmetric case}

The case of nonunity $\tau$ was neglected in [Forti, 2002] the former sections. Now, the model to be examined is extended and by relying on a theorem proposed in a new theorem is given. The method overcomes the problem of noninvertible input/output activation function $f($ ) in Eq. (14), which therefore can be piecewise linear (PWL) or piecewise analytic. Furthermore the theorem proves the stability in case of nonisolated equilibrium points, too. (See Appendix B).

Theorem 2. A 2-layer $C N N$ is completely stable if the template values are

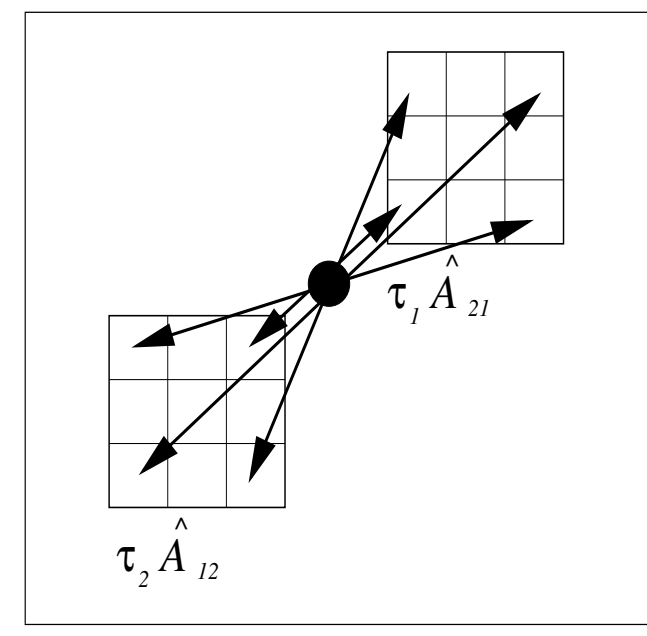

Fig. 4. For nonunity $\tau$ values, $\tau$-cross symmetric rule holds, i.e. weighted template values pointed pairwise by the arrows are the same where the weights are the corresponding time constants.

- central symmetric for intra-layer feedback subtemplates elements $\left(\hat{A}_{11}, \hat{A}_{22}\right)$,

- $\tau$-cross symmetric for inter-layer feedback subtemplates elements $\left(\hat{A}_{12}, \hat{A}_{21}\right)$,

- $f()$ is continuously differentiable, bounded and $f^{\prime}()>0$,

- all equilibrium points are isolated.

Due to the methodology of the proof, the theorem can be extended to the Absolutely Stable class of global pattern formation, similarly to the theorem of M. Forti, which means complete stability for any choice of parameters defining $\hat{A}_{i j}, \hat{B}_{i}$, $\hat{u}_{i}, \hat{z}_{i} i, j \in\{1,2\}$ satisfying the symmetry criteria given above.

The interpretation of the new criterion: Compared to the previous theorem, a modification appears in the $\tau$-cross symmetric criterion which is depicted in Fig. 4 by using the same symbols.

Proof. Let us rearrange the equations of a 2-layer CYCNN

$$
\begin{aligned}
& \tau_{1} \dot{\tilde{x}}_{1}=-\tilde{x}_{1}+\tilde{A}_{11} \tilde{y}_{1}+\tilde{A}_{21} \tilde{y}_{2}+\tilde{B}_{1} \tilde{u}_{1}+\tilde{z}_{1} \\
& \tau_{2} \dot{\tilde{x}}_{2}=-\tilde{x}_{2}+\tilde{A}_{12} \tilde{y}_{1}+\tilde{A}_{22} \tilde{y}_{2}+\tilde{B}_{2} \tilde{u}_{2}+\tilde{z}_{2}
\end{aligned}
$$

as

$$
\begin{aligned}
\dot{\tilde{x}}_{1}= & -\frac{1}{\tau_{1}} \tilde{x}_{1}+\frac{1}{\tau_{1}} \tilde{A}_{11} \tilde{y}_{1}+\frac{1}{\tau_{1}} \tilde{A}_{21} \tilde{y}_{2} \\
& +\frac{1}{\tau_{1}}\left(\tilde{B}_{1} \tilde{u}_{1}+\tilde{z}_{1}\right)
\end{aligned}
$$




$$
\begin{aligned}
\dot{\tilde{x}}_{2}= & -\frac{1}{\tau_{2}} \tilde{x}_{2}+\frac{1}{\tau_{2}} \tilde{A}_{12} \tilde{y}_{1}+\frac{1}{\tau_{2}} \tilde{A}_{22} \tilde{y}_{2} \\
& +\frac{1}{\tau_{2}}\left(\tilde{B}_{2} \tilde{u}_{2}+\tilde{z}_{2}\right)
\end{aligned}
$$

Again, by the concatenation of equations [as in Eq. (8)], one can obtain,

$$
\dot{x}=-\tau x+A^{*} y+B^{*} u+z^{*}
$$

where

$$
A^{*}=\left(\begin{array}{cc}
\frac{1}{\tau_{1}} \tilde{A}_{11} & \frac{1}{\tau_{1}} \tilde{A}_{21} \\
\frac{1}{\tau_{2}} \tilde{A}_{12} & \frac{1}{\tau_{2}} \tilde{A}_{22}
\end{array}\right), \quad B^{*}=\left(\begin{array}{cc}
\frac{1}{\tau_{1}} \tilde{B}_{1} & 0 \\
0 & \frac{1}{\tau_{2}} \tilde{B}_{2}
\end{array}\right)
$$

and

$$
z^{*}=\left(\begin{array}{l}
\frac{1}{\tau_{1}} \tilde{z}_{1} \\
\frac{1}{\tau_{2}} \tilde{z}_{2}
\end{array}\right), \quad \tau=\left(\begin{array}{cc}
\tau_{1} \cdot I & 0 \\
0 & \tau_{2} \cdot I
\end{array}\right) .
$$

Consequently, we obtained original CYCNN equation again with the exception of matrix $\tau$. The Lyapunov function introduced in Appendix B implies strict symmetry requirement on $A^{*}$. This requirement implies symmetric $\hat{A}_{11}$ and $\hat{A}_{22}$ which is the same as in the hypothesis of the previous theorem. Hence, compared to the previous theorem, the only modification this matrix $\tau$ multiplication leads to can be written as

$$
\left(\frac{1}{\tau_{1}} \tilde{A}_{21}\right)^{T}=\frac{1}{\tau_{2}} \tilde{A}_{12}
$$

or

$$
\tau_{2}\left(\hat{A}_{12}\right)_{k l}=\tau_{1}\left(\hat{A}_{21}\right)_{-k-l},
$$

hich is the $\tau$-cross symmetric rule. This rule is depicted in Fig. 4.

\subsection{Nonsymmetric case}

Being armed with the new formalism, another Lyapunov function can be rewritten, which again implies a specific relation of intra- and inter-layer template values. This case can be regarded as an extension of the case given in [Gilli, 1993] which considers single-layer networks only.

\section{Theorem 3. A 2-layer $C N N$ is completely stable if}

- the constant ratio rule holds for intra-layer feedback sub-template elements $\left(\hat{A}_{11}, \hat{A}_{22}\right)$,
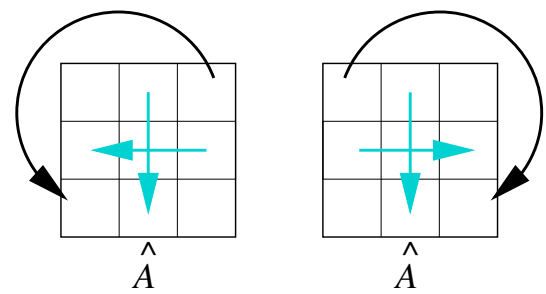

Fig. 5. The constant ratio rule of template elements holds if the ratio of the northern and southern template elements (vertical gray arrows) multiplied by the ratio of the eastern and western elements (horizontal gray arrows) is equal to the ratio of north-southern and south-western elements (black arrow). Or in short $\left(a_{n e} / a_{s w}\right)=\left(a_{n} / a_{s}\right)\left(a_{e} / a_{w}\right)$ and $\left(a_{n w} / a_{s e}\right)=\left(a_{n} / a_{s}\right)\left(a_{w} / a_{e}\right)$. In case of a 2-Layer CNN (2LCNN), intra-layer feedbacks (i.e. $\hat{A}_{11}$ and $\left.\hat{A}_{22}\right)$ must possess this property.

- the constant ratio rule holds between inter-layer feedback sub-template elements $\left(\hat{A}_{12}, \hat{A}_{21}\right)$,

- zero additive term $(\hat{B} * \hat{u}+\hat{z}=0)$ is present,

- layer time constants are unity $\left(\tau_{1}=\tau_{2}=1\right)$,

- $f()$ is continuously differentiable, bounded and $f^{\prime}()>0$

- all equilibrium points are isolated.

The interpretation of the criteria. The so-called constant ratio rule is a term for the rule specified by M. Gilli et al. It explains the stability criterion as a ratio of template elements. We say a template conforms to the rule, if the ratio of the northern and southern template elements multiplied by the ratio of the eastern and western elements is equal to the ratio of north-southern and south-western elements (see Fig. 5 for explanation). Similarly, in a $2 \mathrm{LCNN}$, the same rule must hold for intra-layer feedback sub-template elements, too.

With the same symbolism used in Fig. 5, the constant ratio rule between the two layers is displayed in Fig. 6 which is articulated in terms of the inter-layer feedback sub-template elements.

The example below satisfies the criteria of the theorem in question:
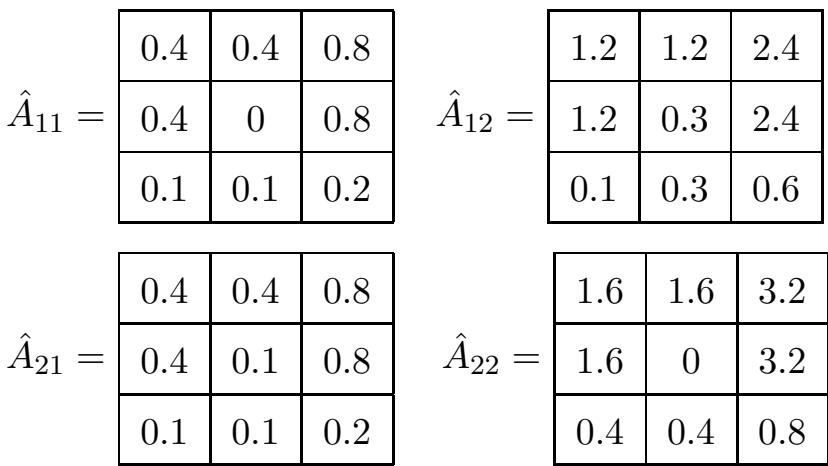

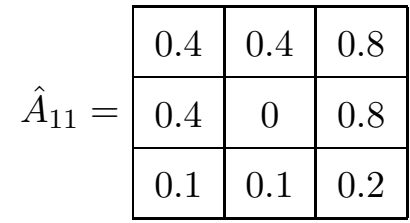




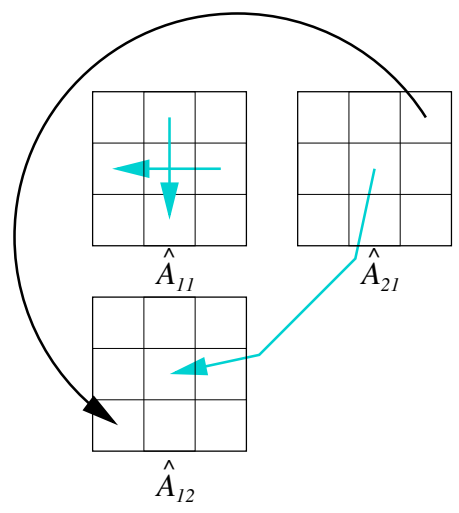

(a) Eq. (20)

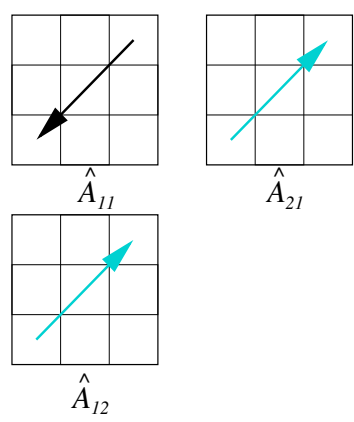

(b) Eq. (21)

Fig. 6. The required constant ratio rule between inter-layer feedback template elements.

Proof. Assume a Lyapunov function in the following quadratic form,

$$
V(x)=-\frac{1}{2} y^{T} P y
$$

where $P=D(A-I)$ is symmetric and consists of non-negative entries only. If there exists a $D=$ $\operatorname{diag}\left\{d_{1}, d_{2}, \ldots, d_{M N}\right\}$ diagonal matrix which satisfies the symmetry criterion for $P$, the network is completely stable. (For the proof, see Appendix C.)

Let us introduce a formalism,

$$
a_{i j}= \begin{cases}(A)_{i j}-1, & \text { if } i=j \\ (A)_{i j}, & \text { else. }\end{cases}
$$

From the diagonality assumption on $D$, one can write $P_{i k}=d_{i} a_{i k}$, and from the symmetricity assumption on $P, P_{i k}=P_{k i} \Leftrightarrow d_{i} a_{i k}=d_{k} a_{k i}$.

From the principle of spatial invariance we have a Toeplitz type restriction on matrix $A$ [see Fig. 2(b)]. The neighboring elements of $D$ must obey the following relation,

$$
\frac{d_{i}}{d_{i+1}}=\frac{a_{i+1, i}}{a_{i, i+1}}=\frac{a_{e}}{a_{w}} .
$$

Consequently, every $N$ th neighboring elements are in relation

$$
\frac{d_{i}}{d_{i+N}}=\frac{a_{i+N, i}}{a_{i, i+N}}=\frac{a_{n}}{a_{s}} .
$$

Since the above holds for all $i, d_{i} / d_{i+1}$ constitute a geometrical series along the diagonal of $D$ (i.e. $\left.d_{i} / d_{i+N}=\left(d_{i} / d_{i+1}\right)^{N} \triangleq q^{N}\right)$. As a consequence,

$\frac{d_{i}}{d_{i+N+1}}=\frac{d_{i}}{d_{i+N}} \frac{d_{i}}{d_{i+1}}=\frac{a_{i+N+1, i}}{a_{i, i+N+1}}=\frac{\mathbf{a}_{\mathbf{n e}}}{\mathbf{a}_{\mathbf{s w}}}=\frac{\mathbf{a}_{\mathbf{n}}}{\mathbf{a}_{\mathbf{s}}} \frac{\mathbf{a}_{\mathbf{e}}}{\mathbf{a}_{\mathbf{w}}}$ $\frac{d_{i}}{d_{i+N-1}}=\frac{d_{i}}{d_{i+N}} \frac{d_{i}}{d_{i-1}}=\frac{a_{i+N-1, i}}{a_{i, i+N-1}}=\frac{\mathbf{a}_{\mathbf{n w}}}{\mathbf{a}_{\mathbf{s e}}}=\frac{\mathbf{a}_{\mathbf{n}}}{\mathbf{a}_{\mathbf{s}}} \frac{\mathbf{a}_{\mathbf{w}}}{\mathbf{a}_{\mathbf{e}}}$.
These relations describe the constant ratio rule. By writing $A$ instead of $\tilde{A}$ (i.e. extension from one single layer to double layer), the consequences for 2LCNN can be examined.

The same rule applies for $A_{11}$ independently of the other submatrix element in the hypermatrix. Having a geometrical series in $\tilde{D}=$ $\left\{d 1, \ldots, d_{M N}, d_{M N+1}, \ldots, d_{2 M N}\right\}$, it leads to the following similar, but much trickier rules,

$$
\frac{d_{i}}{d_{i+M N}}=\frac{a_{i+M N, i}}{a_{i, i+M N}}=\frac{a_{c}^{21}}{a_{c}^{12}} .
$$

Consequently,

$$
\begin{aligned}
\frac{d_{i}}{d_{i+N+M N}} & =\frac{d_{i}}{d_{i+N}} \frac{d_{i}}{d_{i+M N}}=\frac{a_{i+N+M N, i}}{a_{i, i+N+M N}} \\
& =\frac{\mathbf{a}_{\mathbf{n}}^{\mathbf{2 1}}}{\mathbf{a}_{\mathbf{s}}^{\mathbf{1 2}}}=\frac{\mathbf{a}_{\mathbf{n}}^{\mathbf{1 1}}}{\mathbf{a}_{\mathbf{s}}^{\mathbf{1 1}}} \frac{\mathbf{a}_{\mathbf{c}}^{\mathbf{1 2}}}{d_{i+1+M N}} \\
& =\frac{d_{i}}{d_{i+1}} \frac{d_{i}}{d_{i+M N}}=\frac{a_{i+1+M N, i}}{a_{i, i+1+M N}} \\
& =\frac{\mathbf{a}_{\mathbf{e}} \mathbf{2 1}}{\mathbf{a}_{\mathbf{w}}^{\mathbf{1 2}}}=\frac{\mathbf{a}_{\mathbf{e}}^{\mathbf{1 1}}}{\mathbf{a}_{\mathbf{w}}^{\mathbf{1 1}}} \frac{\mathbf{a}_{\mathbf{c}}^{\mathbf{2 1}}}{\mathbf{a}_{\mathbf{c}}^{\mathbf{1 2}}}
\end{aligned}
$$

For diagonal compass points (i.e. southwestern, south-eastern, north-western, northeastern),

$$
\begin{aligned}
\frac{d_{i}}{d_{i+N+1+M N}} & =\frac{d_{i}}{d_{i+1}} \frac{d_{i}}{d_{i+N}} \frac{d_{i}}{d_{i+M N}}=\frac{a_{i+N+1+M N, i}}{a_{i, i+N+1+M N}} \\
& =\frac{\mathbf{a}_{\mathbf{n e}}^{\mathbf{2 1}}}{\mathbf{a}_{\mathbf{s w}}^{\mathbf{1 2}}}=\frac{a_{n}^{11}}{a_{s}^{11}} \frac{a_{e}^{11}}{a_{w}^{11}} \frac{a_{c}^{21}}{a_{c}^{12}}=\frac{\mathbf{a}_{\mathbf{n e}}^{\mathbf{1 1}}}{\mathbf{a}_{\mathbf{c}}^{\mathbf{1 1}}} \frac{\mathbf{a}_{\mathbf{c}}^{\mathbf{1 2}}}{}
\end{aligned}
$$




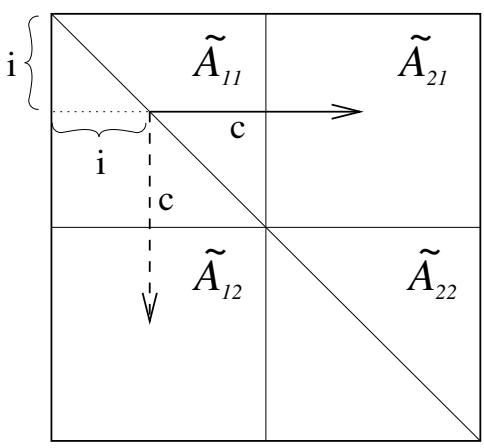

(a) Eq. (22)

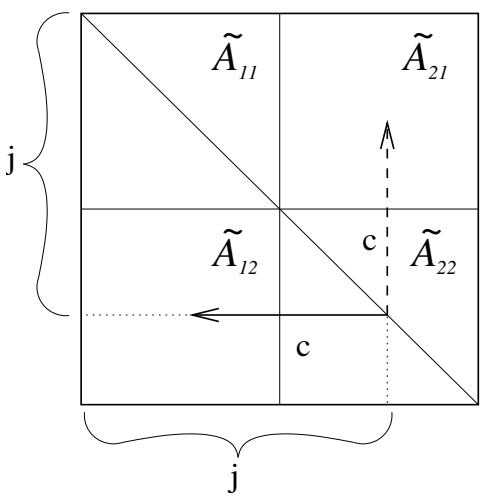

(b) Eq. (23)

Fig. 7. The ratio of the elements pointed to by arrows in matrix $(\tilde{A})$ are in relations that are described by the corresponding equations.

$$
\begin{aligned}
\frac{d_{i}}{d_{i+N-1+M N}} & =\frac{d_{i}}{d_{i-1}} \frac{d_{i}}{d_{i+N}} \frac{d_{i}}{d_{i+M N}}=\frac{a_{i+N-1+M N, i}}{a_{i, i+N-1+M N}} \\
& =\frac{\mathbf{a}_{\mathbf{n w}}^{\mathbf{2 1}}}{\mathbf{a}_{\mathbf{s e}}^{\mathbf{1 2}}}=\frac{a_{n}^{11}}{a_{s}^{11}} \frac{a_{w}^{11}}{a_{e}^{11}} \frac{a_{c}^{21}}{a_{c}^{12}}=\frac{\mathbf{a}_{\mathbf{n w}}^{\mathbf{1 1}}}{\mathbf{a}_{\mathbf{s e}}^{\mathbf{1 1}}} \frac{\mathbf{a}_{\mathbf{c}}^{\mathbf{1 2}}}{}
\end{aligned}
$$

The relation of Eq. (20) is depicted in Fig. 6(a). By similar considerations one can get,

$$
\frac{a_{s e}^{21}}{a_{n w}^{12}}=\frac{a_{s e}^{11}}{a_{n w}^{11}} \frac{a_{c}^{21}}{a_{c}^{12}} \quad \frac{a_{s w}^{21}}{a_{n e}^{12}}=\frac{a_{s w}^{11}}{a_{n e}^{11}} \frac{a_{c}^{21}}{a_{c}^{12}} .
$$

And from similar rearrangements, it is easy to show that

$$
\begin{aligned}
& \frac{a_{s e}^{21} a_{s e}^{12}}{a_{n w}^{21} a_{n w}^{12}}=\frac{\mathbf{a}_{\mathbf{n}}^{11}}{\mathbf{a}_{\mathbf{s}}^{\mathbf{1 1}}} \frac{\mathbf{a}_{\mathbf{w}}^{\mathbf{1 1}}}{\mathbf{a}_{\mathbf{e}}^{\mathbf{1 1}}}=\frac{\mathbf{a}_{\mathbf{n w}}^{\mathbf{1 1}}}{\mathbf{a}_{\mathbf{s e}}^{\mathbf{1 1}}} \\
& \frac{a_{s w}^{21} a_{s w}^{12}}{a_{n e}^{21} a_{n e}^{12}}=\frac{\mathbf{a}_{\mathbf{n}}^{11}}{\mathbf{a}_{\mathbf{s}}^{\mathbf{1 1}}} \frac{\mathbf{a}_{\mathbf{e}}^{\mathbf{1 1}}}{\mathbf{a}_{\mathbf{w}}^{\mathbf{1 1}}}=\frac{\mathbf{a}_{\mathbf{n e}}^{\mathbf{1 1}}}{\mathbf{a}_{\mathbf{s w}}^{\mathbf{1 1}}}
\end{aligned}
$$

which can be depicted as in Fig. 6(b).

By examining the Block Toeplitz structure of $A$, one can conclude that the spatial invariance criterion that lead to the conclusions above, do not imply any relation between $d_{M N}$ and $d_{M N+1}$. This would be particularly interesting so as to conclude whether $D$ can be divided into two parts in which two independent geometrical series are formed or if it must form a single one.

To answer this controversial question, let us write what we used in the deduction of the rules above a bit more formally. The first step [Eq. (19)] can be written as

$$
\frac{d_{i}}{d_{i+c}}=\frac{a_{i+c, i}}{a_{i, i+c}}=q^{c},
$$

which must hold regardless of the following substitution $(j=i+c)$,

$$
\frac{d_{j-c}}{d_{j}}=\frac{a_{j, j-c}}{a_{j-c, j}}=q^{c} .
$$

Roughly, if $c \geq M N$ then Eq. (22) [see Fig. 7(a)] leads to Eqs. (21) and (20), while Eq. (23) [see Fig. 7(b)] implies symmetric rules

$$
\frac{a_{n e}^{21}}{a_{s w}^{12}}=\frac{a_{n e}^{22}}{a_{s w}^{22}} \frac{a_{c}^{21}}{a_{c}^{12}},
$$

which completes the constant ratio rule in general.

\section{4. $\tau$-Nonsymmetric case}

Similarly to the theorem in Sec. 4.2, we here examine the effect of using nonunity layer time constants $(\tau)$, summarized in the following theorem as,

Theorem 4. Theorem 3 holds for any ratio of layer time constants.

Theorems which define a larger than zero set measure of region of parameters in which stability holds could only have importance in engineering practice. From this aspect, Theorem 4 significantly contributes to Theorem 3 .

Proof. A Lyapunov function similar to Eq. (17) is used, but here $H=\left(A^{*}-\tau\right)$ which modifies Eq. (18) as,

$$
a_{i j}= \begin{cases}\left(A^{*}\right)_{i j}-(\tau)_{i i}, & \text { if } i=j \\ \left(A^{*}\right)_{i j}, & \text { else } .\end{cases}
$$




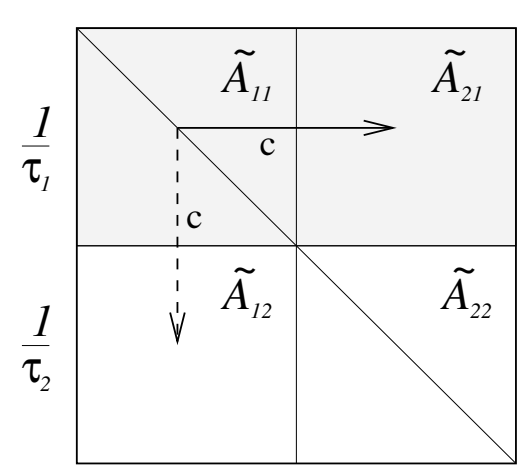

Fig. 8. The matrix $A^{*}$ first used in Eq. (15) and later in Eq. (24) has this structure. Its submatrices are multiplied by $1 / \tau_{1}$ and $1 / \tau_{2}$ where they are gray and white, respectively. In the proof of Theorem 4 this matrix must be symmetrized by multiplying it by $D$ in order to satisfy the corresponding hypothesis.

Let us map matrix elements as $a^{i j *} \rightarrow a^{i j}$ as

$$
\begin{array}{ll}
a^{11 *}=\frac{1}{\tau_{1}} a^{11} & a^{21 *}=\frac{1}{\tau_{1}} a^{21} \\
a^{12 *}=\frac{1}{\tau_{2}} a^{12} & a^{22 *}=\frac{1}{\tau_{2}} a^{22} .
\end{array}
$$

Hence, the rule above transforms Eq. (20) to

$$
\begin{aligned}
\frac{a_{n e}^{21 *}}{a_{s w}^{12 *}} & =\frac{a_{n e}^{11 *}}{a_{s w}^{11 *}} \frac{a_{c}^{21 *}}{a_{c}^{12 *}}=\frac{\frac{1}{\tau_{1}} a_{n e}^{21}}{\frac{1}{\tau_{2}} a_{s w}^{12}}=\frac{\frac{1}{\tau_{1}} a_{n e}^{11}}{\frac{1}{\tau_{1}} a_{s w}^{11}} \frac{\frac{1}{\tau_{1}} a_{c}^{21}}{\frac{1}{\tau_{2}} a_{c}^{12}} \\
& =\frac{\mathbf{a}_{\mathbf{n e}}^{\mathbf{2 1}}}{\mathbf{a}_{\mathbf{s w}}^{\mathbf{1 2}}}=\frac{\mathbf{a}_{\mathbf{n e}}^{11}}{\mathbf{a}_{\mathbf{s w}}^{\mathbf{1 1}}} \frac{\mathbf{a}_{\mathbf{c}}^{\mathbf{2 1}}}{\mathbf{a}_{\mathbf{c}}^{\mathbf{1 2}}}
\end{aligned}
$$

which means that the mapping does not change the original statements.

The reason is simply as follows: during the mapping, $\tilde{A}$ is modified as in Fig. 8 and depending on the magnitude of $c$, this leads to two distinct cases in the equations which can be derived from the symmetry criterion on matrix $P^{*}$ :

(1) The $1<c<M N$ case leads to rules, where the matrix elements referred to as $a_{i, i+c}$ and $a_{i+c, i}$ are in the same hypermatrix partition, so their ratio will not change by multiplying the elements of the partition by an arbitrary nonzero constant (i.e. $a_{i, i+c}^{*} / a_{i+c, i}^{*}=a_{i, i+c} / a_{i+c, i}$ ).

(2) The $M N<c<2 M N$ case leads to rules which simplify the ratio exactly the same way as in Eq. (26).

Hence, one can see that $\tau$ values always simplify, which validates the theorem.

\subsection{Sign symmetric case}

The theorem introduced in [Chua \& Roska, 1990] and later used in [Chua \& Wu, 1992], prescribes the sign relation of template elements rather than the perfect relation of template values, which defines a much larger class of stable templates. Now, these findings are generalized to multi-layers.

Theorem 5. A $2 L C N N$ is stable almost everywhere if,

- intra-layer feedbacks $\left(\hat{A}_{11}, \hat{A}_{22}\right)$ are strictly signsymmetric (see Fig. 10)

$$
\begin{aligned}
& \operatorname{sgn}\left(\hat{A}_{k k}\right)_{i j}=(-1)^{i+j} \text { or } \\
& \operatorname{sgn}\left(\hat{A}_{k k}\right)_{i j}=(-1)^{i} \text { or } \\
& \operatorname{sgn}\left(\hat{A}_{k k}\right)_{i j}=(-1)^{j} \text { or } \\
& \operatorname{sgn}\left(\hat{A}_{k k}\right)_{i j}=(-1)^{0} \quad \forall k \in\{1,2\}
\end{aligned}
$$

- the sign of both of the inter-layer feedbacks are equal to that of the intra-layer feedbacks or its inverse (see Fig. 11)

$\operatorname{sgn}\left(\hat{A}_{k, 3-k}\right)=-\operatorname{sgn}\left(\hat{A}_{k k}\right)$ or

$\operatorname{sgn}\left(\hat{A}_{k, 3-k}\right)=\operatorname{sgn}\left(\hat{A}_{k k}\right)$.

The following template is an arbitrary example for the first template group in the second row of Fig. 11.
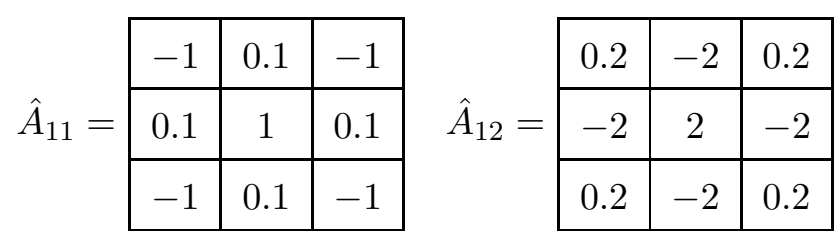

$$
\hat{A}_{21}=\begin{array}{|c|c|c|}
\hline 0.3 & -3 & 0.3 \\
\hline-3 & 3 & -3 \\
\hline 0.3 & -3 & 0.3 \\
\hline
\end{array}
$$

$\hat{A}_{22}=$\begin{tabular}{|c|c|c|}
\hline-4 & 0.4 & -4 \\
\hline 0.4 & 4 & 0.4 \\
\hline-4 & 0.4 & -4 \\
\hline
\end{tabular}

Convergence almost everywhere is a weaker condition than complete stability because it can refer to unstable cases like equilibrium points, limit cycles and chaotic trajectories, though only pathologically. The consequences define a much wider class of solutions than the previous theorems (say, a class of set of nonisolated solutions), which makes these theorems suitable for engineering purposes.

Definition 5. A dynamical system is stable almost everywhere if the set that does not converge to a 
constant equilibrium point has a Lebesque measure zero $^{3}$ (see [Chua \& Wu, 1992])

In the proof of Theorem 5 we will generalize the stability criterion given in [Chua \& Wu, 1992], following Hirsch's theorem in [Hirsch, 1985] claimed for cooperative/competitive systems.

Definition 6. A dynamical system $\dot{x}=F(x), F \in$ $C^{1}$ is cooperative if

$$
\frac{\partial F_{i}(x)}{\partial x_{j}} \geq 0 \quad \forall i \neq j
$$

which means that the Jacobian matrix possesses the non-negative off-diagonals only property at every location.

Definition 7. A dynamical system $\dot{x}=F(x), F \in$ $C^{1}$ is irreducible if a sequence of indexes from $i$ to $j$ exists $\left(\exists\left\{n_{1}=i, n_{2}, n_{3}, \ldots, n_{m-1}, n_{m}=j\right\}\right)$ such that

$$
\Delta F(x)_{n_{l}, n_{l-1}} \neq 0, \quad \forall i, j
$$

where $l \in\{2, \ldots, m\}$ or equivalently, $\exists P$ permutation matrix such that, $P \Delta F(x) P^{T}=\left(\begin{array}{cc}X & Y \\ 0 & Z\end{array}\right)$.

Let us consider it in case of a $2 \mathrm{LCNN}$,

$$
\frac{\partial F(x)_{i}}{\partial x_{j}}=(A)_{i j} \frac{\partial f_{i}(x)}{\partial(x)_{i}}
$$

where

$$
\dot{x}=F(x)=-x+A y+B u+z .
$$

By finding a suitable transformation, our dynamical system can be transformed into a cooperative system. A solution is given by changing variables $x \rightarrow J x^{\prime}$, where $J$ is chosen to be $\operatorname{diag}\left\{(-1)^{n_{1}},(-1)^{n_{2}}, \ldots,(-1)^{n_{M N}}\right\}$ and $n_{i} \in$ $(0,1)$. Consequently, we get

$$
J \dot{x}^{\prime}=-J x^{\prime}+A f\left(J x^{\prime}\right)+B u+z \mid J^{-1},
$$

$\dot{x}^{\prime}=F^{\prime}\left(x^{\prime}\right)=-x^{\prime}+J^{-1} A f\left(J x^{\prime}\right)+J^{-1} B u+J^{-1} z$.

As a consequence the Jacobian matrix of $F^{\prime}$ is

$$
\Delta F^{\prime}\left(x^{\prime}\right)=J^{-1} A \Delta f\left(J x^{\prime}\right) J-I
$$

where $\Delta f()_{i j}=\delta_{i j} f_{i}^{\prime}()$ and $I=\delta_{i j}$. Since they are diagonal, they commute and by a $A^{\prime}=J^{-1} A J$ substitution one can get,

$\Delta F^{\prime}\left(x^{\prime}\right)=J^{-1} A J \Delta f\left(J x^{\prime}\right)-I=A^{\prime} \Delta f\left(J x^{\prime}\right)-I$.

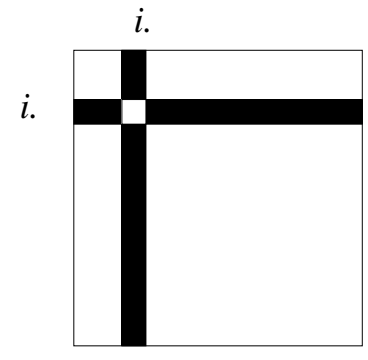

$A^{\prime}$

Fig. 9. The sign inversion of $A$ under a $J A J$ transformation caused by a single negative entry (i.e. $(-1)$ ) in the diagonal of matrix $J$ at the $i$ th position, where black regions refer to the sign-inverted part of the matrix.

Note that $J=J^{-1}$ which will ease the considerations later. The Jacobian matrix at every offdiagonal is,

$$
\frac{\partial F_{i}^{\prime}\left(x^{\prime}\right)}{\partial x_{j}^{\prime}}=\left.\left(A^{\prime}\right)_{i j} \frac{d f(v)}{d v}\right|_{\left(J x^{\prime}\right)_{j}} .
$$

If $A^{\prime}$ is irreducible, non-negative at every offdiagonal position, the system is stable almost everywhere. Hence, we are looking for all appropriate $J$ matrices, which might lead to an irreducible $A^{\prime}$ with non-negative off-diagonals. The task is now to find a methodology that generates all the possible solutions.

Note that irreducibility is intact under a $J A J$ affine transformation.

In general, rewriting an ODE equation into a compact form as it was done in case of the CNN (in Eq. (9), resulting in a matrix $A$ ), will not code all the constraints that the original system (i.e. CNN) had. These constraints include the principle of $s p a-$ tial invariance or diverse type of boundary conditions. As long as the principle of spatial invariance considerably decreases the degree of freedom of the matrix, the later constraint may alter only the structure of the Block Toeplitz matrix.

As a result of this investigation, we present all the possible solutions where Dirichlet boundary condition is assumed.

Every $J_{i i}=(-1)$ will result in a sign inversion of the corresponding (i.e. $i$ th) column and (i.e. $i$ th) row as depicted in Fig. 9.

Matrix $A$ is not only Toeplitz but also sparse, which means that nonzero entries are located exclusively at diagonal and codiagonal positions of the

\footnotetext{
${ }^{3}$ The definition of stable almost everywhere is used in Hirsch sense [Hirsch, 1985].
} 
diagonal and codiagonal blocks of the matrix. In the case of a $2 \mathrm{LCNN}$, the same applies to the intralayer feedback and the inter-layer feedback blocks in the hypermatrix. For a template size larger than $3 \times 3$, co-diagonals are filled up to the $r$ th order.

First, for the sake of simplicity, we collected the solutions for a CNN of size $M \times N$. In order to get spatial invariant solutions, realize that invariant codiagonals must appear in the matrix $A$. In order to get this structure in $A$, we must have a periodic sign series in $J$ (i.e. periodic $n_{i}$, too). The periodicity may be zero, unity, $N$ or mixture of them (call it a composite later) which results in diverse but invariant, sign inverted blocks in matrix $A$.

One can conclude that only a few possibilities remain for $J$ :

- Identity cases: $n_{1}=0, n_{2}=0, \ldots, n_{M N}=0 \Rightarrow$ $J=\{-1,-1, \ldots,-1\}$ which is equivalent to $n_{1}=$ $1, n_{2}=1, \ldots, n_{M N}=1 \Rightarrow J=\{1,1, \ldots, 1\}$ since both projects $A$ onto $A^{\prime}$ without any effect;

- Alternating cases: $J=\left\{1,-1,1, \ldots,(-1)^{M N}\right\}$ or $J=\operatorname{diag}\left\{-1,1,-1, \ldots,(-1)^{M N}\right\}$ leads to chess table sign arrangement in $A^{\prime}$ which correspond to the vertically striped structures in the template;

- $N$ block cases: $J=\operatorname{diag}\{\underbrace{1,1, \ldots, 1}, \underbrace{-1,-1, \ldots,-1}$ $\left.1,1, \ldots, 1, \ldots,(-1)^{M-1}\right\}$ and its inverse $J=$ $\operatorname{diag}\{\underbrace{-1,-1, \ldots,-1}, \underbrace{1,1, \ldots, 1}, \underbrace{-1,-1, \ldots,-1}$, $\left.\ldots,(-1)^{M}\right\}$ where multiple series of ones and minus ones follow each other in blocks of $N$ s. These $J_{\mathrm{S}}$ produce chess table arrangements in the $A^{\prime}$ as well but with square sizes of $N$. These cases correspond to the horizonally striped structures in the template;

- Composite of block $\mathrm{N}$ and alternating cases: Any multiplication of the alternating cases and the block cases leads to new solutions. These cases form the class of stable templates with chess table sign patterns.

The systematic sign-inversions in $A$ correspond to sign inversions at the template sign patterns. We can visualize the rules above. If the sign pattern of a template matches to one of the sign patterns depicted in Fig. 10, the template belongs to the class of stable almost everywhere templates.

The transformation of the found configurations of $J_{\mathrm{S}}$ to the case of $2 \mathrm{LCNN}$ leads to a larger set of stable template sign patterns which are given as follows.

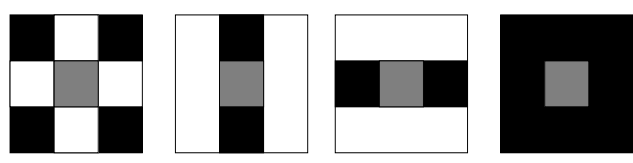

Fig. 10. These are the template sign symmetries of the spatially invariant solutions of $(J A J)_{i j} \geq 0$, where $J_{i j}=$ $(-1)^{n_{k}} \delta_{i j}$. The black regions refer to the sign-inverted part of the template. Non-negative, non-positive and arbitrary values are at black positions, white positions and gray positions, respectively.

The use of two independent layers $\left(\hat{A}_{12}=0\right.$ and $\hat{A}_{21}=0$ ) recalls the Single-Layer CNNs' cases and implies the stability criteria specified above. Interesting questions arise whenever the two layers interconnect via nonzero inter-layer feedback templates.

While seeking for all possible configurations of $J_{\mathrm{s}}$ of $2 \mathrm{LCNNs}$, we will point out that one more case exists which multiplies the number of solutions of those found in the case of the Single-Layer CNNs' class.

- $M N$ block case: $n_{1}=0, n_{2}=0, \ldots n_{M N}=0$, $n_{M N+1}=1, n_{M N+2}=1, \ldots n_{2 M N}=1$ or its inverse. Both have the same influence. They result in an inversion on the off-diagonal matrices in hypermatrix $A$ which corresponds to a sign inversion in the inter-layer feedbacks.

Next, for the generalization of the solutions given above, the parity of the CNNs size (MN) has to be fixed, since it affects the sign inversion patterns for inter-layer feedbacks $\left(A_{i j} i \neq j\right)$.

As long as the even parity case prescribes the same patterns for both inter-layer feedbacks $\left(\hat{A}_{21}, \hat{A}_{12}\right)$ and for the intra-layer feedback $\left(\hat{A}_{11}, \hat{A}_{22}\right)$ patterns, the odd case inverts the sign patterns of the inter-layer feedbacks.

It may be easier to describe the sign inversions in $J$ as a composition of waves. (e.g. $\left.\sum_{j} \operatorname{sgn}\left(\sin \left(\omega_{j} i\right)\right)\right)$.

Considering the question above in this frame, we can describe it as a phase shift between the two halves of $J_{\mathrm{S}}$ or an addition of a new wave the sum in the odd case. The wavelength of this wave should be $2 \mathrm{MN}$.

Remark 3. The mixture of inversions where the first half of matrix $J$ follows one pattern (or periodicity $\omega_{j}$ ), while the other half follows another, does not lead to spatially invariant patterns in $\tilde{A}_{12}$ and in $\tilde{A}_{21}$, consequently they cannot result in a realizable 


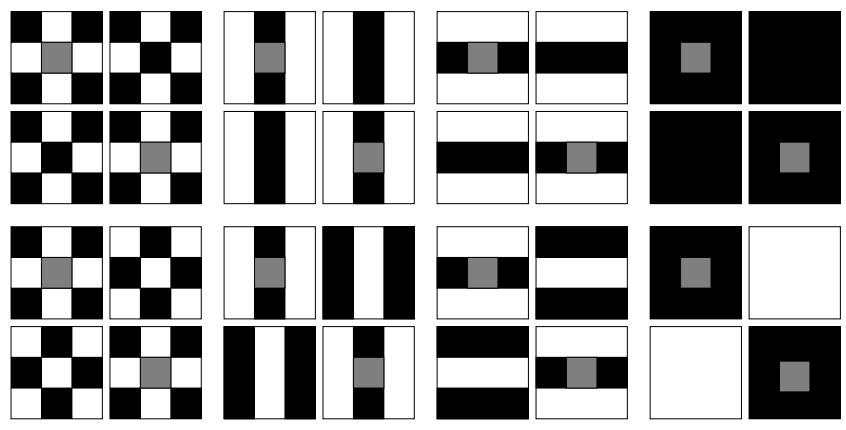

Fig. 11. The set of stable sign patterns. 2 by 2 groups refer to the intra- and inter-layer feedback templates' signs. Within each group, the templates are arranged in the following order: $\left(A_{11} A_{21} ; A_{12} A_{22}\right)$.

\begin{tabular}{|c|c|c|}
\hline- & 0 & + \\
\hline 0 & $\mathrm{~A}$ & 0 \\
\hline+ & 0 & - \\
\hline
\end{tabular}

\begin{tabular}{|c|c|c|}
\hline+ & 0 & - \\
\hline 0 & $\mathrm{~A}$ & 0 \\
\hline- & 0 & + \\
\hline
\end{tabular}

Fig. 12. Two sign patterns from the class of stable templates.

inter-layer feedback template. For this reason, these types of patterns must be excluded.

Remark 4. The class of stable sign patterns which can be generated from the considerations above results in templates that follow the same sign periodicity in both intra-layer and inter-layer feedback templates. Note that, they can be inverses of each other.

However, the odd case of $M N$ inverts our claim for the inter-layer feedback, the parity of the size itself has no effect on this stability criterion at all. (See Fig. 11.)

Remark 5. So far we have not considered the role of the layer time constants $(\tau)$ in this theorem with respect to its unity or nonunity. In fact, there is no need for any consideration since time constants can be only positive, hence no modification can have an effect on this theorem.

\subsubsection{Invariant topologies}

In [Chua \& Roska, 2002] we can find two more solutions (see Fig. 12) that are hard to prove with the given formalism, however, can be generated by topologically invariant transformations.

Considering the topological structure of the network of what these patterns seemingly assume, equivalence may be found between the displayed patterns and the sparse but sign symmetric CNNs (see Fig. 13).

Without mentioning all the possible combinations of topologically invariant transformations applied on CNN and alike networks (see [Radványi, 2000], the rotated, but sparse version of stable patterns that can be obtained by the trick presented above are displayed in Fig. 14.

\subsubsection{Sign patterns for larger templates}

In the former sections, we restricted ourselves merely to double-layer architectures (2LCNN) with $3 \times 3$ template size. By assuming larger template sizes, the matrix responsible for describing dynamics of the CNN in the first role will gradually lose its relative sparseness, hence it would complicate the deduction of most rules summarized in the theorems above. Due to the simplicity of the application of Hirsch's theorems and the wide range usability of the matrix concatenation technique in Eq. (10), theorems may be extended in the direction of even more layers and larger template sizes, resulting in rules which are still not too complicated to check against the templates.

The logic we follow will be roughly that

$$
\begin{aligned}
J A J= & \operatorname{function}(i-j) \Rightarrow J=\operatorname{diag}\left\{(-1)^{n_{1}},\right. \\
& \left.(-1)^{n_{2}},(-1)^{n_{3}}, \ldots,(-1)^{n_{N}}\right\} .
\end{aligned}
$$

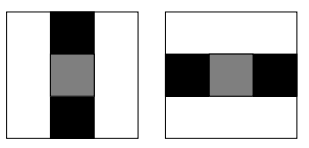

(a) A stable CNN sign-pattern from Fig. 10

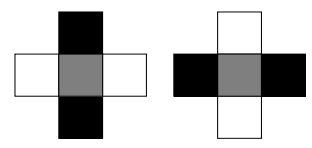

(b) An equivalent but sparse network realization of same sign pattern

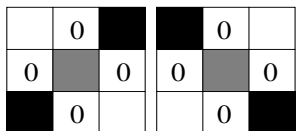

(c) A topologically equivalent network obtained by rotation of (b)

Fig. 13. The steps of transformation required to get the patterns shown in Fig. 12 . 

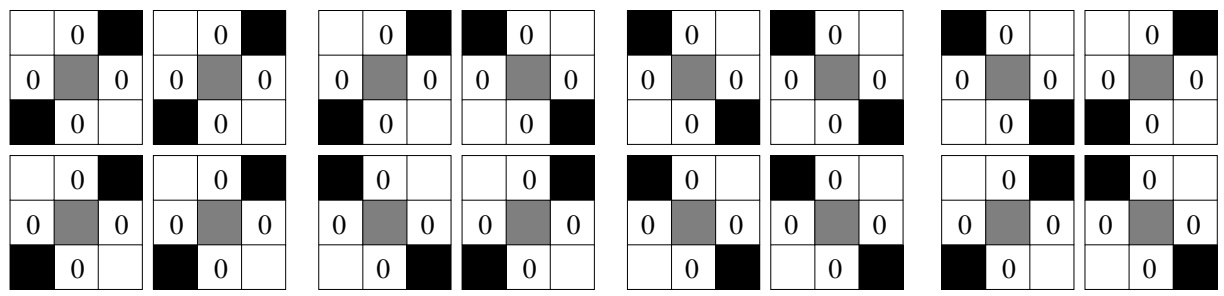

Fig. 14. All sign patterns generated by rules depicted in Fig. 13.

In other words, we seek all solutions of $J_{\mathrm{s}}$ composed of any series of power of $(-1)$ s which result from the Toeplitz, or more precisely Toeplitz like, conditions on JAJ.

Due to the Einstein formalism,

$$
\begin{aligned}
\operatorname{sgn}(J A J) & =\operatorname{sgn}\left(J_{i j} A_{j k} J_{k l}\right) \\
& =\operatorname{sgn}\left(\delta_{i j} J_{i} A_{j k} \delta_{k l} J_{l}\right)=J_{i} J_{l}=\zeta \otimes \zeta^{T}
\end{aligned}
$$

where vector $\zeta^{T}=\left[J_{1}, J_{2}, \ldots, J_{n}\right]$ and $\otimes$ symbolizes the diadic multiplication operator.

Since $J_{i} J_{l}=J_{l} J_{i}, \zeta \otimes \zeta^{T}$ is always symmetric, hence Eq. (28) may be written as,

$$
\zeta \otimes \zeta^{T}=\operatorname{sgn}(\text { function }(|i-j|))
$$

Definition 8. A matrix with a chess board type sign pattern is defined as a matrix composed of rectangles of the same sign (zero included) from which none of these rectangles are in contact with another of the same sign.

Lemma 1. There exists a constructive way to determine $\zeta$ from $\zeta \otimes \zeta^{T}$, if and only if $\zeta \otimes \zeta^{T}$ is sign symmetric, has positive diagonal and has chess board type signs exclusively.
No proof is required.

Only symmetric chess board type sign patterns in $\zeta \otimes \zeta^{T}$ satisfy the left-hand side of Eq. (29) since any sign inversion in $J$ causes sign inversion in the corresponding row and column at one time. Only diagonal sign patterns satisfy the right-hand side of the same equation. The two patterns that meet both statements above are the homogeneous one and the chess board signed one which are produced by constant (e.g. $J=\operatorname{diag}\{1,1,1, \ldots, 1\}$ ) or alternating series (e.g. $J=\operatorname{diag}\{-1,1,-1, \ldots, 1\}$ ), respectively, or their inverse (already shown in Sec. 4.5).

Note that these special chess board types of matrices resemble chess boards since the used rectangle size is unity.

To be more precise, one can realize that the constraint (i.e. right-hand side of Eq. (29) above is applied for a single-layer one-dimensional network exclusively where the matrix $A$ is Toeplitz.

Concluding remarks on non-one-dimensional structures involves Block Toeplitz matrices which extend the degree of freedom of $\zeta$ and of $J$ as

$$
\zeta \otimes \zeta^{T}=\operatorname{sgn}(\text { function }(\overbrace{|i-j|}^{\text {horizontal dependency }}, \overbrace{|[i / N]-[j / N]|}^{\text {vertical dependency }}))
$$

where [.] denotes the integer truncation. The terms $[i / N],[j / N]$ refer to partitions in $A$ that are needed to have homogeneous sign field.

Indeed, the searched unknown function( ) is depending on $[i / N]-[j / N]$, which explains Toeplitz-like sign alterations just like it was required for matrix elements in the one-dimensional case. This leads to the case that has already been shown in Sec. 4.5.

Continuing this methodology toward two-layers, one might write the MN block case as

$$
\zeta \otimes \zeta^{T}=\operatorname{sgn}(\text { function }(|i-j|,|[i / N]-[j / N]|,|[i / M N]-[j / M N]|))
$$

which points much further in a constructive way.

For example the alternating and the $N$ block alternating cases correspond to the alternating signs in templates in horizontal or in vertical directions that can be expanded for larger than $3 \times 3$ templates, like $5 \times 5$. 


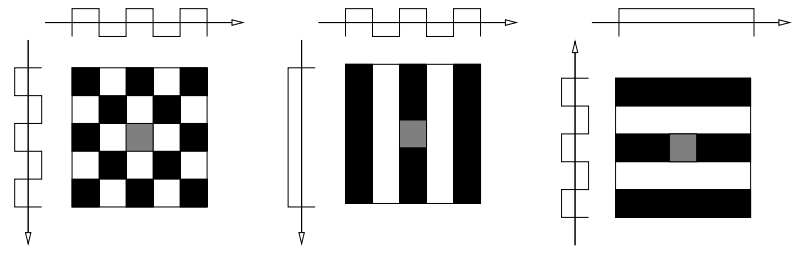

Fig. 15. Sign alteration frequencies of $5 \times 5$ templates are depicted. On the left-hand side, both the horizontal and the vertical sign alteration frequency are equal to one. In the center, horizontal sign alteration frequency is equal to one while vertical sign alteration frequency is zero. The one on the right-hand side is just opposite of the previous one.

Later we will see Eq. (30) is only a special case which holds for two-layers only but not for larger networks.

Due to the concatenation technique introduced in Sec. 3, as the degree of freedom of function() gradually increases, the number of solutions for $J$ multiplies.

Now, we can claim our next theorem.

Theorem 6. A 2LCNN with an arbitrary template size is stable almost everywhere if,

- the horizontal sign alteration frequency in all the templates are equal to one or zero up to the exception of the intra-layer feedback's central element,

- the vertical sign alteration frequency in all templates are equal to one or zero up to the exception of the intra-layer feedback's central element,

- the phase of the alteration are equal in groups $\operatorname{sgn}\left(\hat{A}_{11}\right)=\operatorname{sgn}\left(\hat{A}_{22}\right)$ and $\operatorname{sgn}\left(\hat{A}_{12}\right)=\operatorname{sgn}\left(\hat{A}_{21}\right)$ but may vary between groups.

For better understanding the expression alteration frequency see Fig. 15. above,

Below an example is given for the theorem

$\hat{A}_{11}=$\begin{tabular}{|l|l|l|l|l|}
\hline-1 & 1 & -1 & 1 & -1 \\
\hline-1 & 1 & -1 & 2 & -1 \\
\hline-1 & 1 & 0.1 & 3 & -1 \\
\hline-1 & 1 & -1 & 4 & -1 \\
\hline-1 & 2 & -3 & 4 & -5 \\
\hline
\end{tabular}

$\hat{A}_{12}=$\begin{tabular}{|l|l|l|l|l|}
\hline 1 & -1 & 1 & -1 & 1 \\
\hline 1 & -1 & 1 & -2 & 1 \\
\hline 1 & -1 & 1 & -3 & 1 \\
\hline 1 & -1 & 1 & -4 & 1 \\
\hline 1 & -2 & 3 & -4 & 5 \\
\hline
\end{tabular}

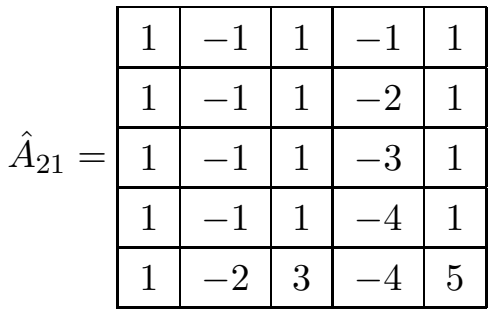

$\hat{A}_{22}=$\begin{tabular}{|c|c|c|c|c|}
\hline-1 & 1 & -1 & 1 & -1 \\
\hline-1 & 1 & -1 & 2 & -1 \\
\hline-1 & 1 & -0.1 & 3 & -1 \\
\hline-1 & 1 & -1 & 4 & -1 \\
\hline-1 & 2 & -3 & 4 & -5 \\
\hline
\end{tabular}

\subsubsection{Sign patterns for more layers}

Considering it for more than two layers, the Toeplitz constraint does not hold as in Eq. (30) but we can write as,

$$
\begin{aligned}
\zeta \otimes \zeta^{T}= & \operatorname{sgn}(\text { function }(|i-j|, \mid[i / N] \\
& -[j / N]|,|[i / M N]|,|[j / M N] \mid))
\end{aligned}
$$

The reason is that, the interaction of cells $i$ and $j$ must be fixed and be a function of the difference of the indexes (i.e. spatially invariant) as long as $i$ and $j$ are located in the same layer, the interaction of cells from the layer $[i / M N]$ and $[j / M N]$ may vary from layer-to-layer. For example, the interaction of the first layer to the second must not be the same as the second layer to the third one. According to the given equation, they must not even be in a kind of sign-symmetric relation.

In relation to the identified multi-layer criteria, the left-hand side of Eq. (31) may be rewritten as

$$
J^{L} \odot\left(\zeta_{1} \otimes \zeta_{1}^{T}\right)=\zeta \otimes \zeta^{T}
$$

where $\odot$ denotes the Kronecker matrix multiplication of a "small" sign modifier matrix $J^{L}$ and the single-layer interaction sign-matrix $\left(\zeta_{1} \otimes \zeta_{1}^{T}\right)$.

For example in a three-layer case, the sign modifier matrix may be

$$
J^{L}=\left(\begin{array}{rrr}
1 & -1 & -1 \\
-1 & 1 & 1 \\
-1 & 1 & 1
\end{array}\right)
$$

which explains that the interaction between layers 1,2 and 1,3 are in a kind of competitive (negative feedback) relation, while all the rest are in a kind of cooperative (positive feedback) relation (for details see [Hirsch, 1985]. 
Finally, summarizing this finding in a theorem:

Theorem 7. A CNN with arbitrary number of layers and with arbitrary but fixed template size is stable almost everywhere if,

- the horizontal sign alteration frequency in all the templates are equal to one or zero up to the exception of the intra-layer feedback's central element,

- the vertical sign alteration frequency in all templates are equal to one or zero up to the exception of the intra-layer feedback's central element,

- sign modifier matrix is chess board type, symmetric and positive diagonal (see Lemma 1).

\subsection{Nonreciprocal case}

Definition 9. When the template values of a CNN are sign-anti-symmetric, the CNN is called nonreciprocal.

In [Chua \& Roska, 1990] the complete stability is conjectured and support nonreciprocal CNNs. The studied dynamics of opposite sign valued template system cover a column-wise (or row-wise) coupling of cells exclusively, which means dynamics are row/column-wise independent of each other, thus it is called one-dimensional CNN or 1DCNN. For the summary, see Appendix E.

Based upon very similar considerations on the structure of the Jacobian matrix, a new conjecture is given, which extends the former stability theorem (complete stability conjecture) to a column-wise (or row-wise) coupled 1D CACE architecture.

Definition 10. A one-dimensional CACE is composed of two inter-connected one-dimensional CNNs with intra-layer feedback of neighborhood radius one, and with scalar inter-layer feedback.

Conjecture 1. A 1DCACE, will always settle in a saturated region and converge to stable equilibrium point if

- inter-layer feedbacks are sign anti-symmetric,

- intra-layer feedbacks are sign anti-symmetric,

- the central element of the intra-layer feedbacks is greater than one $\left(A_{k k}\right)_{c}>1$.

This means the stability of a nonreciprocal CACE architecture.
Pattern Evolution on Layer \#1 in a 1D CACE

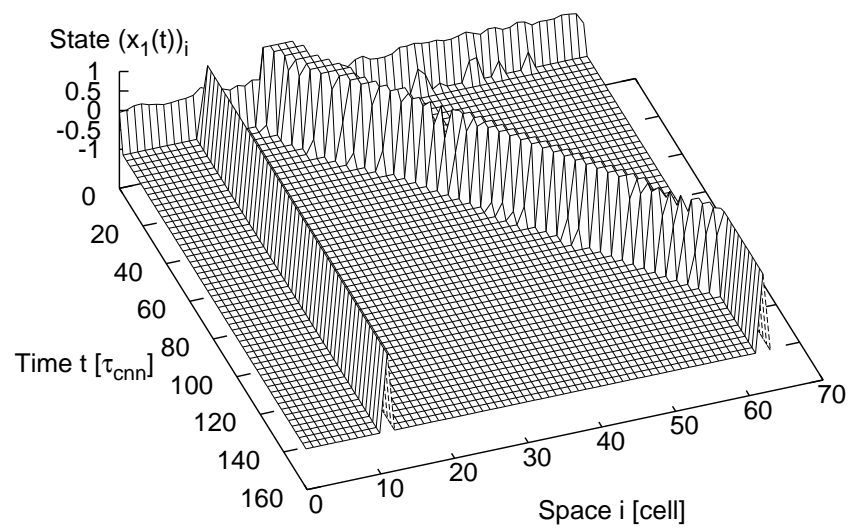

Fig. 16. The time evolution of the one-dimensional CACE structure with a nonreciprocal template.

The interpretation of the criteria: The criteria above defines the following template structure:

$$
\begin{aligned}
& \hat{A}_{11}=\begin{array}{|l|l|l|}
\hline a & b & -c
\end{array} \quad \hat{A}_{12}=e \\
& \hat{A}_{21}=h \quad \hat{A}_{22}=\begin{array}{|l|l|l|}
\hline j & k & -l \\
\hline
\end{array}
\end{aligned}
$$

where $a, b, c, j, k, l>0$ and $e \cdot h<0$.

Similarly to the Single-Layer 1D CNNs case, if any cell $(k)$ reaches the saturated region $(\beta)$, it will generate $\mathrm{a}-1$ at $k$ th (or at $(k+M L)$-th if it is on the second layer) diagonal position in the concatenated Jacobian matrix, and zeros above and below. A concatenated Jacobian matrix is constructed in a way similar to that of matrix $A$ and is constructed from $\hat{A}$ in Eq. (10). Having zeros in a column with the exception of the diagonal, the corresponding eigenvalue can be explicitly given $(=-1)$ and the system can be reduced by a factor of one by neglecting the corresponding row and column. Chua et al. [Chua \& Roska, 1990] found a recursive method to calculate the determinant of the remaining band matrix $(\tilde{A})$, consequently they could point out that only positive eigenvalues exist in the reduced system. This implies that at least one cell will eventually reach the saturated region again from which it never returns.

A similar consideration applies to the CACE case, but no generative solution like this has been found so far for determinant calculation which is inevitable for a system of arbitrary size. Instead, we confirmed all possibilities by a symbolic software package and we claim:

For having positive principal minors of all possible configurations with an assumption on $\hat{A}_{11}$ and 
$\hat{A}_{22}$ as given above, a noncontradictory solution may exist if and only if $\hat{A}_{21}$ and $\hat{A}_{12}$ are of opposite sign scalars.

The example below generates the trajectory in Fig. 16.

$$
\begin{gathered}
\hat{A}_{11}=\begin{array}{l|l|l|}
\hline 1 & 2.5 & -1
\end{array} \quad \hat{A}_{12}=-1 \\
\hat{A}_{21}=1
\end{gathered} \quad \hat{A}_{22}=\begin{array}{ll|l|l|}
\hline 1 & 2.5 & -1 \\
\hline
\end{array}
$$

\section{Conclusion}

A description of a complex cell CNN-UM architecture (CACE) along with the MLCNN is given. This motivated a survey on the possibilities of generalization of stability theorems from SingleLayer architectures to Multi-Layer architectures.

The power of a new formalism was demonstrated by claiming several theorems and one conjecture on multi-layer architectures inherently including the CACE architecture.

By means of constructed new Lyapunov functions the layer time constants dependence of the theorems was made possible.

The inference of the formalism developed has proved to be useful in generalization of many theorems and Lyapunov functions. Its consequences are not completely explored, therefore, it is worth carrying out further investigations in the class of multilayer neural architectures.

\section{Acknowledgments}

Thanks are due to Marco Gilli and Ákos Zarándy for their advice and fruitful ideas. This work has been supported by their advice and the Hungarian Academy of Sciences and the Grant No. 00014-0210884 .

\section{References}

Carmona, G. R., Jiménez Garrido, F., Domínguez Castro, R., Espejo Meana, S. \& Rodríguez Vázquez, A. [2002] CACE1K Users Guide, Instituto de Microelectrónica de Sevilla.

Chua, L. O. \& Yang, L. [1988a] "Cellular neural networks: Theory," IEEE Trans. Circuits Syst. 35, $1257-1272$.

Chua, L. O. \& Yang, L. [1988b] "Cellular neural networks: Theory," IEEE Trans. Circuits Syst. 35, 1273-1290.
Chua, L. O. \& Roska, T. [1990] "Stability of a class of nonreciprocal cellular neural networks," IEEE Trans. Circuits Syst. 37, 1520-1527.

Chua, L. O. \& Shi, B. E. [1991] "Multiple layer cellular neural networks: A tutorial," Algorithms and Parallel VLSI Architectures, eds. Deprettere, E. F. \& van der Veen, A. (Elsevier Science Publishers B.V., Amsterdam), pp. 137-168.

Chua, L. O. \& Wu, C. W. [1992] "On the universe of stable cellular neural networks," Int. J. Circ. Th. Appl. 20, 497-518.

Chua, L. O. \& Roska, T. [2002] Cellular Neural Networks and Visual Computing, Foundations and Applications (Cambridge University Press), Chap. 8, pp. 205-231.

Cortino, F. \& Gilli, M. [2003] "Comparision between the dynamic behavior of Chua-Yang and full-range cellular neural networks," Int. J. Circ. Th. Appl. 31, 423-441.

Espejo, S., Rodríguez-Vázquez, A. Dominíguez-Vázquez, R. \& Carmona, R. [1994] "Convergence and stability of the FSR CNN model," Proc. IEEE Int. Workshop on Cellular Neural Networks and Their Applications, Rome, pp. 411-416.

Forti, M., Liberatore, A. Manetti, S. \& Marini, M. [1993] "Global asymptotic stability for a class of nonsymmetric neural networks," Proc. IEEE Int. Symp. Circuit and System, Chicago, Vol. 4, pp. 2580-2583.

Forti, M. [2002] "Some extension of a new method to analyze complete stability of neural networks," IEEE Trans. Neural Networks 13, 1230-1238.

Gilli, M. [1993] "A Lyapunov functional approach to the study of the stability of cellular neural networks," Proc. IEEE Int. Symp. Circuit and System, Chicago, Vol. 4, pp. 2584-2587.

Hirsch, M. W. [1985] "System of differential equations that are competitive or cooperative II: Convergence almost everywhere," SIAM J. Math Anal. 16, 423-439.

Hopfield, J. J. [1984] "Neurons with graded response have collective computational properties like those of two-state neurons," in Proc. Nat. Acad. Sci. 81, 3088-3092.

Kosko, B. [1988] "Bi-directional associative memory," IEEE Trans. Syst. Manag. Cyb. 18, 49-60.

LaSalle, J. P. [1967] "An invariant principle in the theory of stability," in Differential Equations and Dynamical Systems, eds. Halle, J. K. \& LaSalle, J. P. (Academic Press), pp. 277-286.

Radványi, A. [2000] "On the rectangular grid representation of general CNN networks," Proc. 6th IEEE Int. Workshop Cellular Neural Networks and Their Applications, Catania Piscataway, pp. 387-393.

Rekeczky, Cs., Serrano, T., Roska, T. \& RodríguezVázquez, A. [2000] "A stored program 2nd order/3layer complex cell CNN-UM," Proc. 6th IEEE Int. Workshop on Cellular Neural Networks and Their Applications, Catania Piscataway, pp. 213-218. 


\section{Appendix A}

Proof for Lyapunov Function in Theorem 1 [Chua \& Roska, 2002] (rewritten here).

$$
\begin{aligned}
V(x)= & \sum_{i=1}^{2 M N}\left[\int_{\theta}^{y_{i}} f^{-1}(v) d v\right] \\
& -\frac{1}{2} y^{T} A y-y^{T} B u-y^{T} z
\end{aligned}
$$

Since $A$ is positive definite,

$$
\begin{aligned}
\dot{V}(x)= & \sum_{i=1}^{2 M N} f^{-1}\left((y)_{i}\right)(\dot{y})_{i} \\
& -\dot{y}^{T} A y-\dot{y}^{T} B u-\dot{y}^{T} z
\end{aligned}
$$

By substituting $f^{-1}(y)=x$,

$$
\begin{aligned}
\dot{V}(x) & =\dot{y} x-\dot{y}^{T} A y-\dot{y}^{T} B u-\dot{y}^{T} z \\
& =-\dot{y}^{T}(-x+A y+B u+z) \\
& =-\dot{y}^{T} \dot{x}
\end{aligned}
$$

By $\Delta f(x) \equiv \operatorname{diag}\left\{f^{\prime}\left((x)_{1}\right), f^{\prime}\left((x)_{2}\right), \ldots, f^{\prime}\left((x)_{2 M N}\right)\right\}$ one can write $\dot{y}=\Delta f(x) \dot{x}$, which results in

$$
\begin{gathered}
\dot{V}(x)=-\dot{x}^{T} \Delta f(x) \dot{x} \\
\text { Since } \Delta f(x)_{i j}>0 \forall i, j \Rightarrow \dot{V}(x)<0 \text {. Q.E.D }
\end{gathered}
$$

\section{Appendix B}

In the proof of Theorem 2 we relied on the stability of Eq. (15).

Definition 11. A diagonal mapping $H \in \mathcal{P}$ if and only if for $i=1, \ldots, N, h_{i}\left(x_{i}\right): \mathbb{R} \rightarrow \mathbb{R}$ is a continuous, nondecreasing bounded PWL function such that $h_{i}(0)=0$.

In [Forti, 2002] a fundamentally new approach is used for proving the theorem in which the author claims that a neural network $(N)$ written in the form,

$$
\dot{x}=-D x+A h(x)+c
$$

is completely stable if $h \in \mathcal{P}$, where $D$ is diagonal, $c$ is an arbitrary constant and $A$ is symmetric. The trajectory limit length theory which Mauro Forti et al. develops ensures stability even in cases where nonlinearity $(h)$ is totally not invertible caused by piecewise linear (PWL) horizontal sections. These horizontal sections lead to degenerate cases (where the infinitely many nonisolated equilibrium points exist) hence, only nonstrict Lyapunov functions can be given. A Lyapunov function like this involves the analysis by LaSalle's invariant sets principle that may be rather complicated.

These horizontal sections a play crucial role in increasing the networks learning capability (Vapnik-Chervonenkis dimension) compared to sigmoid $(\tanh ())$ like neural input/output activation functions. As the number of these horizontal sections increase, the pattern formation capability increases too, which simply enriches the family of stable patterns.

The following global Lyapunov function Eq. (B.2) is associated with Eq. (B.1) that overcomes the problem of the assumption of invertible $f($ ). It was assumed in Appendix A, too, indeed it is rooted in the methodology of [Hopfield, 1984].

$$
\begin{aligned}
V(x)= & -\frac{1}{2} h^{T}(x) A h(x)-h^{T}(x) c \\
& +\sum_{i=1}^{N} D_{i} \int_{0}^{x_{i}} \rho \frac{d h_{i}}{d \rho} d \rho
\end{aligned}
$$

which holds for all the affine systems, too.

The steps of the proof are recalled from [Forti, 2002] with variables remapped to our system and with standard Chua-Yang nonlinearity $(f())$ applied instead of $(h(x))$ as,

(1) A nonstrict global energy function is introduced that overcomes the inversion of the noninvertible (PWL) input/output activation function,

$$
\begin{aligned}
V(x)= & -\frac{1}{2} y^{T} A^{*} y-y^{T}\left(B^{*} u+z^{*}\right) \\
& +\sum_{i=1}^{2 M N}(\tau)_{i i} \int_{0}^{x_{i}} \rho \frac{d y_{i}}{d \rho} d \rho .
\end{aligned}
$$

The deduction of $\dot{V}(x) \leq 0$ is, practically, equivalent to the one in Appendix A, but with Dini derivatives at nondifferentiable points of $f()$.

(2) The existence of a function like this is exploited to prove that the total length of the trajectory

$$
L\left(y_{\infty}\right)=\lim _{t \rightarrow \infty} \int_{0}^{t}\|\dot{y}(\sigma)\|_{2} d \sigma<\infty
$$

is finite which means that $y(t)$ must be convergent, well.

(3) which implies the convergence of $x(t)$, as Q.E.D 


\section{Appendix $\mathrm{C}$}

A typical quadratic Lyapunov function [Eq. (C.1)] is assumed in Theorem 3 coming from [Gilli, 1993] and the proof is rewritten here with slight modifications.

$$
V(x)=-\frac{1}{2} y^{T} P y .
$$

We rely on the same Lyapunov function, where the existence of a $D$ matrix is hypothesed which will symmetrize $H=(A-I)$ ( $I$ refers to the identity matrix) in order to get a symmetric $P$ (i.e. $P=$ $\left.D H=H^{T} D^{T}\right)$, positive definite matrix. By $\Delta f(x) \equiv \operatorname{diag}\left\{f^{\prime}\left((x)_{1}\right), f^{\prime}\left((x)_{2}\right), \ldots, f^{\prime}\left((x)_{2 M N}\right)\right\}$ one can write

$$
\begin{aligned}
\dot{V}(x) & =-y^{T} P \dot{y} \\
& =-y^{T} P \Delta f(x) \dot{x} \\
& =-y^{T} P \Delta f(x)(-x+A y) .
\end{aligned}
$$

Because $\Delta f(x) x=\Delta f(x) y$, by substitution,

$$
\begin{aligned}
\dot{V}(x) & =-y^{T} P \Delta f(x)(-y+A y) \\
& =-y^{T} P \Delta f(x)(A-I) y \\
& =-y^{T} P \Delta f(x) H y \\
& =-y^{T} H^{T} D^{T} \Delta f(x) H y \\
& =-y^{T} H^{T}\left(D^{T} \Delta f(x)\right) H y
\end{aligned}
$$

Since $\left(D^{T} \Delta f(x)\right)_{i j}>0 \forall i, j \Rightarrow \dot{V}(x)<0 \quad$ Q.E.D

Remark 6. In contrast to the original theorem, where $D$ is assumed to be diagonal, we found that this matrix property is not required to be met, at all. However, it can be proved that only Toeplitz matrices may appear in such a role but resulting in no extra constraints on the structure of matrix $A$. Consequently it would make no sense to complicate the calculations with these cases.

\section{Appendix D}

In the proof of Theorem 4 the quadratic Lyapunov function may be written as,

$$
V(x)=-\frac{1}{2} y^{T} P y
$$

associated with the nonunity $\tau$ system with nonsymmetric templates. Hence with assuming symmetric $P=D H$ where $H=(A-\tau)$, similar to that of in Appendix C, equations develop as,

$$
\begin{aligned}
\dot{V}(x) & =-y^{T} P \dot{y}=-y^{T} P \Delta f(x) \dot{x} \\
& =-y^{T} P \Delta f(x)(-\tau x+A y) \\
& =-y^{T} P \Delta f(x)(-\tau y+A y) \\
& =-y^{T} P \Delta f(x)(A-\tau) y \\
& =-y^{T} H^{T}\left(D^{T} \Delta f(x)\right) H y
\end{aligned}
$$

Since $\left(D^{T} \Delta f(x)\right)_{i j}>0 \forall i, j \Rightarrow \dot{V}(x)<0$

Q.E.D

\section{Appendix E}

During the support of Conjecture 1 we relied on the complete stability of a nonreciprocal 1D CNN in [Chua \& Roska, 1990]. In this case matrix $A$ will have the following structure,

$$
\hat{A}=\left(\begin{array}{ccc}
0 & 0 & 0 \\
s & p & -s \\
0 & 0 & 0
\end{array}\right) \quad \tilde{A}=\left[\begin{array}{ccccc}
p & -s & & & \\
s & p & -s & & \\
& s & \ddots & \ddots & \\
& & \ddots & & -s \\
& & & s & p
\end{array}\right]
$$

These types of matrices $(\tilde{A})$ belong to the class $P$, which possess the unique property that all of its principle minors are strictly positive as well as the real part of their eigenvalues.

For similar reasons, simple cases when all cells are either in the linear region $(\alpha)$ or in saturated region $(\beta)$, lead to a Jacobian matrix with either positive eigenvalues $(\Delta f(x)=\tilde{A}-I)$ only, or -1 eigenvalues $(\Delta f(x)=-I)$ only, respectively. While the calculation of the principal minors in the latter case is obvious, the first one can be done by an elegant recursive rule proposed by Chua et al.

Eigenvalue calculation when a single cell is in region $\beta$ and the rest is in region $\alpha$, may be decomposed to the above cases, which enables the author to conclude the following remarks:

Remark 7. Regarding the trajectories of anti signsymmetric CNNs,

- no trajectory can settle outside the saturated region,

- any trajectory in the saturated region converges to a stable equilibrium point inside the same region,

- all trajectory in the linear region tends to leave the region.

This supports the conjecture of complete stability. 